\title{
STELLUNG DES GRIECHISCHEN RECHTS IN DER ANTIKEN RECHTSGESCHICHTE
}

\section{Joachim Hengstl ${ }^{*}$}

1 Betrachtet man das Entstehen, Blühen und Vergehen antiker Kulturen, deren Kontakte untereinander und den damit verbundenen kulturellen Austausch sowie die Vielfalt der wechselseitigen Einflüsse, so drängt sich unter juristischem Blickwinkel schnell der Eindruck auf, daß an diesem Wechselspiel auch das Recht teilgenommen haben muß. Das Bild eines fließenden und wachsenden Stroms rechtlicher Entwicklung, tradiert von Epoche zu Epoche, über die Kulturgrenzen und über die Zeiten hinweg, vielleicht bis heute wirkend, ist ebenso eindruckvoll wie überwältigend. Der Gedanke einer zusammenhängenden Geschichte wenigstens der antiken Rechtsordnungen ist nicht neu. Vor allem L Wenger hat diese These entwickelt, bereits in seiner Wiener Antrittsvorlesung 1904, „Römische und antike

* Akademischer Oberrat aD, Universität Marburg. Siehe H Barta „Graeca non leguntur"? Zu den Ursprüngen des europäischen Rechts im antiken Griechenland. Ein Beitrag zur Wissenschaftsund Kulturgeschichte des Rechts. Bd 1 (Wiesbaden, 2010) ISBN 978-3-447-06121-6 gr 8; XIX 683 S; 23 Ill; Bd II $/$ II $^{2}$ Archaische Grundlagen (Wiesbaden, 2011) ISBN 978-3-447-06278-7 gr 8; XVIII 765 S mit 12 Abb, bzw ISBN 978-3-447-06587-0 gr 8; XVIII $522 \mathrm{~S}$ mit $8 \mathrm{Abb}-\mathrm{Zu}$ Bd I des Werks vgl ferner (durchweg kritisch) S Günther in (2011) 12 Bryn Mawr Classical Review 61, sowie Th Finkenauer in sehepunkte (2012) 12 Nr 12 [15-12-2012], URL: http://www. sehepunkte.de/2012/12/18091.html. Die Besprechungen der Bände sind nacheinander entstanden. Der Versuch einer Angleichung hätte die Fertigstellung unabsehbar verzögert. Es wird daher um Nachsicht bei stilistischen und inhaltlichen Ungleichheiten gebeten.

\section{UNISA $\cong$}

Fundamina Volume 21 | Number 1 | 2015 pp 33-62
Doi: $10.17159 / 2411-7870 / 2015 / v 21 n 1 a 3$ Print ISSN 1021-545X/ Online ISSN 2411-7870 (C) Unisa Press 
Rechtsgeschichte", ${ }^{1}$ und sein ebenso umfassendes wie umfangreiches Meisterwerk Die Quellen des römischen Rechts ${ }^{2}$ ist von dieser Auffassung geprägt. Andere Rechthistoriker haben aus unterschiedlichen Blickwinkeln den gleichen Gedanken vertreten. Die in der Neuzeit ständig zunehmende Flut an Quellen vor allem aus dem Alltag der verschiedenen antiken Rechtsordnungen scheint die These von Übernahmen und Abhängigkeiten erst recht zu bestätigen. Die Untersuchungen zu den ,internationalen“ kulturellen Beziehungen nehmen gleichfalls $\mathrm{zu}^{3}$ und untermauern den Gedanken, daß das Recht dabei nicht unbeeinflußt geblieben sein kann. ${ }^{4}$ In dieser Weise gestärkt, sollte die Idee eines sich ständig entwickelnden Rechtswesens überzeugen - über die Zeiten hinweg, selbst das Rechtsdenken der Römer und die Institutionen des Römischen Rechts nachhaltig beeinflußend und folglich auch noch heutige Rechtssysteme zumindest der westlichen Welt. ${ }^{5}$ Der Gedanke einer derartigen, wechselseitigen Beeinflussung in der Rechtsmaterie und einer epochen- und grenzenübergreifenden, stetigen Rechtsentwicklung ist in den letzten Jahrzehnten allerdings nicht mehr artikuliert worden.

Barta, emeritierter Zivilrechtslehrer an der Universität Innsbruck, greift diesen Gedanken auf, erweitert ihn und sucht ihn in einem auf vier Gesamtbände angelegten monumentalen Werk zu verifizieren. Sein Blickwinkel wie seine durchgängig tragende These sind mit dem Titel Graeca non leguntur bestens beschrieben: Man habe die literarische Überlieferung der Griechen nicht hinreichend beachtet und deren rechtsgeschichtliche Tragweite folglich völlig verkannt und übergangen. Das griechische Recht habe zwar nicht als Rechtsordnung des Alltags überlebt, wohl aber hätten griechische Rechtsvorstellungen und Rechtseinrichtungen Einfluß auf und über die römische Epoche hinaus gehabt. Darüber hinaus ist Barta bemüht, Verbindungslinien zwischen den griechischen Rechtsinstitutionen und dem Alten Orient einschließlich des Pharaonischen Ägypten zu ziehen. Sein Unterfangen, die zeitlichen und räumlichen Distanzen zwischen den verschiedenen Kulturen zu überbrücken, ist ebenso anspruchsvoll wie weit gespannt. Eine nicht minder schwierige Aufgabe ist es freilich, ein derart umfangreiches und ehrgeiziges Werk angemessen $\mathrm{zu}$ beschreiben und $\mathrm{zu}$ bewerten, noch dazu, bevor es vollständig vorliegt.

\section{Graz, 1905.}

L Wenger Die Quellen des römischen Rechts (Wien, 1953) passim.

3 Siehe beispielsweise und vor allem W Burkert Die Griechen und der Orient. Von Homer bis zu den Magiern (München, 2003). Das maßgebliche altorientalische Textmaterial findet sich in Umschrift und Übersetzung bei KA Kitchen \& PJN Lawrence Treaty, Law and Covenant in the Ancient Near East 3 Bde Pt 1: The Texts; Pt 2 Text, Notes and Chronograms; Pt 3: Oral Historical Survey (Wiesbaden, 2012).

4 Zum altorientalischen, altägyptischen, jüdischen Recht uam siehe R Westbrook (ed) A History of Ancient Near Eastern Law 2 Bde (Leiden / Boston, 2003).

$5 \quad \mathrm{Vgl}$ dazu beispielsweise R David \& G Grasmann Einführung in die großen Rechtsysteme der Gegenwart. Rechtsvergleichung (München / Berlin, 1966) (Barta bekannt, zB S 191 Anm 830). 
Es erscheint sinnvoll, vorab die rechtshistorischen Gegebenheiten zu resümieren, innerhalb deren sich Bartas Darstellung bewegt. Damit läßt sie sich besser einordnen. Die Graeca, auf die Barta sich bezieht, sind nämlich im Wesentlichen literarische Quellen aus dem klassischen und hellenistischen Athen. Athen war damals Teil der griechischen Polis-Welt, die ihren Ursprung mitsamt all ihren geistigen Strömungen und Auswirkungen im 8 / 7 Jahrh v Chr gehabt hat. Das ist übrigens auch die Zeit der griechischen Kolonisierungsbewegung gewesen. Die Alltagsquellen dieser wie der späteren Zeit bestehen weitgehend aus Inschriften aus den verschiedenen poleis und hernach aus den Papyri des griechisch-römischen Ägypten. Auf dieses Material geht Barta bislang kaum ein.

Das rechtshistorische Umfeld ist rasch skizziert. Die Schrift ist um 3000 v Chr erfunden und alsbald in Ägypten und in Mesopotamien auch zur Aufzeichnung rechtlicher Vorgänge benützt worden. Während Ägypten über die nächsten drei Jahrtausende zumeist isoliert geblieben ist, entwickelten sich in Mesopotamien und seiner Umgebung bis zur Zeitenwende eine Reihe an Reichen, oder diese übernahmen die Herrschaft über mehr oder minder große Regionen. Die Schrift waren in Ägypten die Hieroglyphen, und hieraus wurden die hieratische und von dieser die demotische Schrift abgeleitet. Letztere ist bis in nachchristliche Zeit benutzt und nach kurzer Unterbrechung vom Koptischen abgelöst worden. Man wird nicht fehl gehen, wenn man diese Entwicklung neben Anderem als Manifest ägyptischer Selbstbehauptung ansieht. An Quellen wie an Untersuchungen zum Recht des pharaonischen Ägypten mangelt es nicht, und es liegen sogar Gesamtdarstellungen vor. ${ }^{6}$ All das genügt freilich nicht, um Anhaltspunkte für rechtliche Einflüsse zu bieten, die über die Grenzen Ägyptens hinausreichen. Das ist nicht zuletzt durch die Quellenlage bedingt. Rechtlich relevantes Alltagsmaterial gibt es nämlich in nennenswertem Maße erst aus dem Neuen Reich und nur aus einem einzigen Ort, der Siedlung der Arbeiter an den Königsgräbern bei dem heutigen Deir el-Medineh (Oberägypten). Von hier liegen reichlich 10000 hieratische Ostraka und Papyri vor. ${ }^{7}$ Verallgemeinern lassen sich die Aussagen der aus einer derartigen Siedlung stammenden Texte nicht.

Aus der Zeit vor dem Neuen Reich fehlt es keineswegs an zahlreichen Rechtsquellen. Sie sind jedoch zeitlich, örtlich und inhaltlich zerstreut und nicht leicht heranzuziehen. Das Bild des Justizwesens muß aus Funktionärstiteln

6 Siehe zuletzt SL Lippert Einführung in die altägyptische Rechtsgeschichte (Münster ua, 2008) passim, und vgl dazu T Mrsich „Methodisches zur altägyptischen Rechtsgeschichte“ I, II, in (1913) 130 Zeitschrift der Savigny Stiftung für Rechtsgeschichte Rom Abt S 507-526; (1914) 131 Rom Abt S 349-369.

7 Siehe dazu ua W Helck Die datierten und datierbaren Ostraka, Papyri und Graffiti von Deir el-Medineh, bearbeitet von A Schlott (Wiesbaden, 2002); A Dorn \& T Hofmann (eds) Living and Writing in Deir el-Medine. Socio-historical Embodiment of Deir el-Medine Texts (Basel, 2006); S Allam „Recht im pharaonischen Ägypten“ in Die Rechtskulturen der Antike. Vom Alten Orient bis zum Römischen Reich hrsgg von U Manthe (München, 2003) S 15-54. Vgl ferner <www.unimuenchen.de/dem-online $>$ oder www.lmu.de/dem-online. 
und den diversen Urkunden einer Administration herausgearbeitet werden, die die Gewaltentrennung noch nicht kennt. ${ }^{8}$ Einem Rechtstransfer fehlt damit die Voraussetzung eines umfassend durchgebildeten, vorbildhaften Rechtswesens mit entsprechenden Institutionen. Rechtlich relevantes Material bieten ab der ägyptischen Spätzeit um 700 v Chr die bis ins 2. Jahrh n Chr reichenden demotischen Urkunden. ${ }^{9}$ Von diesem Material an Alltagsurkunden lassen sich gleichfalls keine Einflüsse erwarten, die sich auf andere Rechtsordnungen erstreckt haben könnten. Man wird sie grundsätzlich verneinen dürfen. Sachlich liegt das an der geographischen Isolierung des Pharaonenreiches. Kulturelle und gedankliche Strömungen haben diese Schwelle durchaus überwunden. Sowohl archäologische Funde wie das Alte Testament belegen das. Rechtsvorstellungen aber haben sich schwerlich auf vergleichbare Weise zur Kopie angeboten. An dieser Bewertung ändern weder die „persische Reichsautorisation“ noch die demotischen Rechtsbücher etwas. Unter der „Reichsautorisation“ wird eine ausdrückliche und billigende Anerkennung lokaler Normen unter achämenidischer Herrschaft verstanden. ${ }^{10}$ Sie kann also nur nach Ägypten hinein, nicht aber als Vorlage fremder Rechtsordnungen gewirkt haben. Die demotischen Rechtsbücher belegen zwar ein hohes Maß an Jurisprudenz; ${ }^{11}$ eine über Ägypten hinausreichende Wirkung ist jedoch nicht zu erkennen und auch nicht zu erwarten.

In Mesopotamien und in seiner Umgebung ist die Keilschrift benützt worden. Der Quellenreichtum der rechtlichen Überlieferung in Keilschrift ist hoch, und die Zahl der dadurch belegten Epochen, Reiche und Regionen ist nicht gering. An literarischen oder philosophischen Äußerungen zum Recht mangelt es gänzlich. Die verschiedenen Rechtsordnungen sind lediglich durch Alltagsurkunden, Herrschererlasse; Briefe und Ähnliches mehr dokumentiert. Zu erwähnen sind ferner die keilschriftlichen Rechtssammlungen, deren Gesetzescharakter umstritten ist. ${ }^{12}$ Merkbar sind lokal

8 Siehe beispielhaft A Philip-Stéphan Dire le droit en Égypte pharaonique. Contribution à l'étude des structures et mécanismes juridictionnels jusqu'au Nouvel Empire (Bruxelles, 2008) passim (vgl dazu die eingehende Besprechung von J Hengstl in (2014) 131 Zeitschrift der Savigny Stiftung der Rechtsgeschichte Rom Abt S 424-429).

9 Siehe J Manning „Demotic Law“ in Westbrook (o Anm 4) S 819-862.

10 Hierunter ist der Gedanke an eine ausdrückliche und billigende Anerkennung lokaler Normen zu verstehen, grundlegend P Frei „Zentralgewalt und Lokalautonomie im Achämenidenreich“ in Reichsidee und Reichsorganisation im Perserreich (Freiburg / Göttingen, ${ }^{2} 1996$ ) S 5-31; Dies „Die persische Reichsautorisation. Ein Überblick“ in (1995) 1 Zeitschrift für altorientalisches und biblisches Recht 1-35, jeweils mwN Darauf einzugehen besteht hier kein Anlaß.

11 Siehe SL Lippert Ein demotisches juristisches Lehrbuch. Untersuchungen zu Papyrus Berlin P 23757 rto (Wiesbaden, 2004); Dies aaO (o Anm 6); dazu ferner J Hengstl „Gab es Rechtswissenschaft in der außerrömischen Antike?" in R van den Bergh et al (eds) Libellus ad Thomasium. Essays in Roman Law, Roman-Dutch Law and Legal History in Honour of Philip J Thomas ((2010) 16(1) Fundamina editio specialis) S 164-183 (180-182).

$12 \mathrm{Vgl}$ dazu die Sammlungen in R Haase Die keilschriftlichen Rechtssammlungen in deutscher Fassung (Wiesbaden, ${ }^{2}$ 1979); oder M Roth Law Collections from Mesopotamia and Asia Minor (Atlanta, 1995), siehe ferner Kitchen / Lawrence aaO (o Anm 3). 
oder zeitlich bedingte Eigenheiten. Die Überlieferungslage erlaubt jedoch keine weitergehenden Schlüsse, da nur selten größere Urkundengruppen vorliegen oder sich zusammenfügen lassen. Für die Annahme, irgendwelche keilschriftlich belegten Rechtsinstitute hätten sich über den keilschriftlichen Bereich ausgewirkt, gibt es keinen zweifelsfreien Anhaltspunkt.

Ein Blick muß an dieser Stelle auch der israelitischen Rechtsüberlieferung gelten, die vor allem das Alte Testament enthält. Die Eigenständigkeit des israelitischen Gedankenguts, aber auch die Verflochtenheit mit den Israel umgebenden Hochkulturen ist überdeutlich. Von einem Einfluß israelitischen Rechtsdenkens oder israelitischer Rechtsinstitute auf die Rechtsordnungen der Umgebung oder der nachfolgenden Zeit ist jedoch zumindest bislang nichts festzustellen.

Mit den Rechtszeugnissen des pharaonischen Ägypten, dem keilschriftlichen Bereich und dem im Alten Testament manifesten Judentum liegt ein über 2000 Jahre umfassendes Quellenmaterial rechtlicher Natur vor. Nicht erfasst sind mit diesen umfangreichen Quellenbeständen mannigfache Kultur- und Rechtsordnungen. Von ihnen gibt es nur geringfügige Quellenbestände, die sich aber ständig vermehren. Beispiele hierfür sind nabatäische oder früharabische Zeugnisse.

Dennoch überlappen sich die Zeugnisse der antiken Hochkulturen lediglich in ihren Endphasen mit den Zeugnissen der frühen griechischen und der frühen römischen Rechtsordnung. Die griechische Poliswelt hat ihre Anfänge im 8. und 7. Jahrh v Chr, und das sagenhafte Gründungsdatum Roms ist 753 v Chr. In Mesopotamien herrschte zu dieser Zeit eine Gemengelage. Seit der Mitte des 2. Jahrt v Chr war nämlich die ethnische Gruppe der Aramäer aus der arabischen Wüste allmählich nach Nordsyrien und Mesopotamien vorgedrungen und hatte dort um die Wende zum 1. Jahrt v Chr verschiedene Kleinstaaten gebildet. Das neuassyrische Reich unterwarf diese Kleinstaaten im 9. und 8. Jahr v Chr. Obgleich das Reich bereits damals durch aramäische Deportierte und Einwanderer aramäisch geprägt gewesen ist, und obgleich es auch anderweitige aramäische Zeugnisse gibt, ist es doch nicht möglich, das Bild einer ,,aramäischen Rechtsordnung“ zu entwerfen - es hat sie vermutlich nie gegeben. ${ }^{13}$ Nicht besser ist es um die Einflußmöglichkeiten einer ,ägyptischen Rechtsordnung“ bestellt. In 656 v Chr beginnt die Spätzeit des pharaonischen Ägypten und damit die - bis in das 20. Jh n Chr währende Fremdherrschaft über das Land. Bereits in der vorausgehenden ,3. Zwischenzeit“ (1070-656 v Chr) hat sich Ägyptens politische Lage stark verändert; das Land hatte seine Außenbesitzungen verloren und war in seine eigentlichen geographischen Grenzen zurückgedrängt. Weiterreichende rechtliche Einflüße kann man für die Folgezeit lediglich in zwei Momenten vermuten. Beide sind bereits oben erwähnt worden - die „persische Reichsautorisation“ und die demotischen Rechtsbücher, und beiden ist dort jede außerägyptische Wirkung abgesprochen worden.

$13 \mathrm{Zu}$ den Aramäern siehe vor allem E Lipinski The Aramaeans. Their Ancient History, Culture, Religion (Leuven / Paris, 2000) mwN. 
Diese Feststellung muß auf den ersten Blick erstaunen. An Handelsbeziehungen und kulturellen Einflüssen zwischen den verschiedenen Kulturen und über große Entfernungen hinweg hat es bereits in den vorgeschichtlichen Zeiten nicht gemangelt und erst recht nicht in der Folgezeit. Barta erwähnt und erörtert sie immer wieder, mit weiterführenden Nachweisen. Es ist also unnötig, auf dieses Thema hier weiter einzugehen. Eine andere Frage verlangt aber Beachtung: Weshalb und auf welche Weise sollen Rechtsordnungen unterschiedlicher und zeitlich verschiedener Kulturen auf einander einwirken? Vollends verfehlt und anachronistisch ist es, ein Streben nach Rechtsvereinheitlichung in antiken Rechtsordnungen anzunehmen. ${ }^{14}$

2 Der erste Band von Bartas Werk enthält neben den üblichen Präliminarien und einer „Einleitung“ (S 1-56) lediglich das 1. Kapitel „Perspektiven“ (S 57561), welches in zehn Unterkapitel unterteilt ist: „1. Zum Buchtitel“; „2. Zum Wert humanistischer Bildung“; ,3. Europa und griechisches Recht“; „4. Phasen der römischen Rechtsentwicklung“; ,5. 'Andersheit' der griechischen Rechtskultur?“; „6. Gab es ein 'gemeines’ griechisches Recht?“; „7. Olympische Religion und Heroenkulte“; „8. Rechtskollisionen im archaischen Griechenland“; „9. Anfänge des Völkerrechts“; und „10. Rezeption durch Rom?““.

Richtungsweisende Weichen werden bereits in der Einleitung dargestellt. Vor allem ist es der Begriff ,Rechtsdenken“, den Barta vertritt (S 11-13). ,'Rechtsdenken' umfaßt (für ihn) alle Facetten der Beschäftigung mit dem Recht (S. 11)." Die Fortsetzung definiert Bartas Blickwinkel zweifelsfrei: „(Zum Rechtsdenken) gehört die Rechtspraxis (Gesetzgebung, materielle und formelle Rechtsanwendung aller Art, Vertragspraxis und Kautelarjurisprudenz, Rechtsberatung, rechtspolitische und rechtsethische Diskurse und Dialoge, Gerichtsreden, Urkunden, Schriftsätze) ebenso wie der Beginn einer Rechtstheorie und von Methodenfragen (Lehre von der Rechtsanwendung, Hermeneutik, Lückenfüllung uam.); die Rechtsphilosophie, die Lehre von der Gesetzgebung und den Rechtsquellen ebenso wie das Nachdenken über Billigkeit/Epieikeia und Naturrecht." Ob sich ein heutiger Richter, Rechtsanwalt oder Verwaltungsbeamter auf Grund dieser Definition zum „Rechtsdenker“ geadelt fühlen mag? Bartas Sichtweise ist völlig modernistisch. Das muß kein Schade sein, denn irgendeiner Lupe oder Brille muß sich der heutige Rechtshistoriker bedienen, um vergangene Rechtsordnungen zu ergründen.

Vor dem Hintergrund der weiteren Ausführungen fällt bereits in diesem Abschnitt Bartas vorwiegend deskriptive Vorgehensweise auf. Dem entspricht zudem die Art, in der Barta ständig irgendwelche Phänomene zwar umreißt und ihnen Fortwirkungen beimißt, ohne diese aber weiter zu belegen. Ein Beispiel ist

14 So S 61 bei Anm 226. Vgl dazu J Hengstl „Zur Frage von Rechtsvereinheitlichung im frühaltbabylonischen Mesopotamien und im griechisch-römischen Ägypten - Eine rechtsvergleichende Skizze“ in 3e série (1993) 40 Revue Internationale des Droits de l'Antiquité S 27-55. 
sein Gedanke, „die griechische Dichtung und auch die Geschichtsschreibung (hätten) immer wieder grundlegende Fragen von Recht und Gerechtigkeit aufgegriffen" (S 12). Wohl wahr, aber wie hat sich das auf die jeweilige Rechtsordnung ausgewirkt? ${ }^{15}$ In entsprechender Weise pauschal und der exegetischen Begründung entbehrend sind Bartas Monita, ältere Literatur sei von den neueren Rechtshistorikern weitgehend oder völlig übergangen worden, und letztere hätten sich so deren Sichtweise entzogen. Der Gedanke taucht nicht auf, daß neue Quellen und Methoden vielleicht erst der eigenständigen Sichtung und Auswertung bedürfen, ehe Altes auf einer neuen Basis bewertet und nötigenfalls integriert werden kann. Soweit vorab!

Die „Einleitung“ gibt im Weiteren einen „Kapitelüberblick“(S 13-21), informiert „Über Zitate, Zitierweise, Gliederung“( S 21-25), geht unter dem Titel „Troje ‘Europa und griechisches Recht'“" vor allem mit HJ Wolff ins Gericht (S 25-29), ${ }^{16}$ rügt den Verzicht auf „Neue Disziplinen für Rechtswissenschaft und Rechtsgeschichte“ [S 29-31; zB Soziobiologie: „Eine richtig verstandene Rechtsgeschichte wäre in der Lage (gewesen), rechtliche Probleme der Antike, insbesondere auch Griechenlands, in einen antik-gegenseitigen und einen Bezug zur Gegenwart zu bringen“ (S 30)] und die „Neubewertung des griechischen und orientalischen Einflusses“ (S 31-34) zu berücksichtigen, wobei ,bereits Erkanntes ... systematisch mit dem geltenden Recht in Verbindung zu setzen (wäre)“ (S 33). Eine Auflistung von Topoi, bei denen Barta einen ,interkulturellen Vergleich“ vermißt (zu welchem Zweck bleibt unerörtert) und mehrere „Griechische Rechtsfälle“ bilden den nächsten Abschnitt (S 34-49). Die Schilderung der Fälle ist anekdotisch und trägt zur Rechtsgeschichte so wenig bei wie der Rest der Einleitung.

So viel Worte über die 56 Seiten der Einleitung müssen erstaunen. Die Einleitung ist freilich beispielhaft für Bartas (bisheriges) Vorgehensweise insgesamt. Barta agiert vor allem deskriptiv; er resümiert oder zitiert eine Fülle an Lesefrüchten, die seine Belesenheit erweist und sich in dem umfangreichen Literaturverzeichnis spiegelt. ${ }^{17}$ Das erfolgt nicht ohne Schärfen zu vorgeblichen Versäumnissen der

15 Zum Verhältnis von Dichtung und Recht vgl zB R Schmitz-Wiegand in Handwörterbuch zur deutschen Rechtsgeschichte hrsgg von A Erler / E Kaufmann (Berlin, 1990) Bd 4 Sp 232-249sv „Recht und Dichtung“ (Sp 240ff zu Dichtung als Rechtserkentnisquelle); Dies in Handwörterbuch zur deutschen Rechtsgeschichte hrsgg von A Cordes / H Lück / D Werkmüller / R Schmitz-Wiegand (Berlin, ${ }^{2} 2008$ ) Bd 1 Sp 1034-1943, sv „Dichtung und Recht“(Sp 1035-1038 zu Dichtung und Recht in ihrem Wechselbezug; Sp 1038-1041 zu Dichtung als Rechtserkentnisquelle). Allgemeingültiger Grundgedanke ist dabei, daß Recht kein abgesondertes, vom Alltag fein säuberlich geschiedenes Sonderdasein führt, sondern daß die Rechtsvorstellungen das alltägliche Verhalten und eben auch die literarischen Schöpfungen prägen. Naturgemäß vermag jede geistige Entwicklung wiederum das Rechtsdenken zu beeinflussen, aber das Maß ist jeweils nachzuweisen.

16 Siehe HE Troje Europa und griechisches Recht (Frankfurt, 1971). Trojes Gedanken scheinen für B nach allem den maßgeblichen Leitfaden - darf man sagen „Evangelium“? - auf seinem Weg in die antike Rechtsgeschichte gebildet zu haben.

17 Der ungewöhnlich Umfang des Literaturverzeichnisses dürfte sich daraus erklären, daß Barta alles Gelesene aufführt und nicht nur das Verwendete. 
heutigen antikrechtlichen Disziplin, der Barta die Vernachlässigung sowohl früherer rechtshistorischer Erkenntnisse wie auch neuerer rechtlicher wie außerrechtlicher Methoden vorwirft. Der alte Grundsatz cui bono bleibt dabei völlig außer Betracht. Nirgendwo wird nämlich profund, detailliert und anhand sorgsamer Exegesen sowie ausgeführter Beispielen analysiert, in welcher Weise denn jene Erkenntnismöglichkeiten die aktuelle antikrechtliche Forschung fördern könnten. So anschaulich, wenn auch weitschweifig Bartas Darstellung ist, so gerät sie doch nur zu einer Gesamtschau vor allem der antikrechtlichen Sekundärliteratur. Dieses Beginnen ist von einem letztlich anerkennenswerten Eifer getragen: Barta will mit anachronistischer Beweisführung den Lebenswert der antiken Rechtsgeschichte selbst für das heutige Recht nachweisen. Diese Sichtweise ist verfehlt, denn die antike Rechtsgeschichte ist vorrangig Teil der Geschichte, obgleich ihre Erforschung unbedingt auf juristisch fundierter Methodik beruhen muß. Glaubt man freilich, die Geschichtswissenschaft sei, wie vielleicht mehr oder minder die gesamten Geisteswissenschaften, entbehrlich, weil ökonomisch ineffektiv, so gibt es auch für die antike Rechtsgeschichte keine Existenzberechtigung. Selbst die Rechtsprechung läßt sich, wie der Blick auf das spätrömische „Zitiergesetz“ zeigt, ohne Rücksicht auf den Gehalt ökonomisieren. ${ }^{18}$

Bereits die Einleitung läßt den weiteren Gang der Darstellung erkennen: Barta will keine Geschichte des griechischen Rechts oder dessen Institutionen schreiben, sondern eine Ideengeschichte mit dem Anspruch, die Fortwirkung der geschilderten Ideen nachzuweisen. Bartas Werk ist sehr anschaulich angelegt. Das detaillierte Inhaltsverzeichnis (S XI-XIV) übernimmt einen guten Teil der zahlreichen Zwischentitel; das Abkürzungsverzeichnis (S XV-XVIII) löst auch Abkürzungen auf, die gewöhnlich unbemerkt benützt werden; ${ }^{19} 23$ Abbildungen illustrieren (Verzeichnis S XIX); ein „Glossar“ (S 563-575) erläutert eine Vielzahl an Begriffen; das „Literaturverzeichnis“ (S 578-669) ist fast schon eine Bibliographie zur antiken Rechtsgeschichte geworden;20 der Sachindex (,Stichworte“ S 671-683) umfaßt Personen, Orte und Begriffe.

18 Nach dem $426 \mathrm{n} \mathrm{Chr} \mathrm{von} \mathrm{Valentinian} 3$ erlassenen und von Theodosius 2 in CTh 143 eingefügten so genannten „Zitiergesetz“ durften in Rechtsstreitigkeiten nur noch die Rechtsmeinungen von Papinian, Paulus, Ulpian, Modestinus und Gajus berücksichtigt werden, so zu sagen aus „ökonomischen Gründen“.

19 ZB (auch hier unaufgelöst benützt) ,vgl“ = ,vergleiche“. Nachweis ausgefallen zu „ZÄS“ = Zeitschrift für ägyptische Sprache und Altertumskunde (Berlin).

20 Man vermißt allerdings beispielsweise L Rosetti „Materiali per una storia della letteratura giuridica attica“" in Nomos. Direito e sociedade na Antiguidade Clássica / Derecho y sociedade en la Antigüedad Clàsica ed por DF Leão / L Rosetti / M Céu Fialho (Coimbra / Madrid, 2004) S 5173; E Volterra Diritto Romano e diritti orientali (Napoli, 1983) (Nd d Ausgabe Bologna, 1937); ferner J Hengstl ,Ex oriente lux in der Rechtsgeschichte?“ in Timai J. Triantaphylopoulou (Athen, 2000) S 39-57 (peripher gelegentlich und ablehnend zitiert in BII). Nicht aufgenommen ist ferner beispielsweise H Stilett Montaigne für Lehrer (Frankfurt/M, 2004) [nv] (zit S 96, Anm 385: korr „Stilettt“ $\rightarrow$,Stilett"). Leider finden sich in dem an sich so nützlichen Literaturverzeichnis immer 
Ungeachtet all dieser durchdachten und nützlichen Hilfen ist Bartas Werk jedoch didaktisch, inhaltlich und in der Gestaltung keineswegs gelungen. Bereits in der Einleitung finden sich Exzerpte, die da wie auch in Kapitel 1 die fachgerechte Analyse und die explizite Argumentation ersetzen. Zwar ist es für den Leser bequem, anderweitige Sekundärbelege auf diese Weise an der Hand zu haben, in extensivem Maße dient es jedoch nicht der Übersicht, und vielfach ist es schlicht entbehrlich. Das gilt auch für die Vielzahl an Zwischentiteln, die keineswegs hierarchisch gegliedert sind. Auch hierin ist Bartas Vorgehensweise anekdotisch - Barta offeriert Alles in Allem eine Menge an (Lese- und Gedanken-)Früchten, ohne daraus aber ein gelungenes Kompott zu fertigen.

Graeca non leguntur ist ein Werk, welches bei künftigen Arbeiten zum griechischen und römischen Recht unbedingt herangezogen werden muß. Dafür bürgt bereits das umfangreiche Literatur-Verzeichnis (S 571-669), das fast zu einer Bibliographie zur griechischen Rechtsgeschichte geraten ist. Die Auswertung des reichlich herangezogenen Materials läßt freilich zu wünschen übrig. Die Quellenanalyse unterbleibt weitgehend, und es findet auch keine eingehende Auseinandersetzung mit der Sekundärliteratur statt. Bartas Argumentationsweise ist rein deskriptiv. Sie beschränkt sich im Wesentlichen auf Zitate und Textübernahmen, deren Zusammenstellung offenkundig dank verbindender Sätze hinreichend aussagekräftig sein soll.

Vor allem aber übersieht Barta bei all seinen Versuchen, über Räume und Zeiten hinweg, rechtliche Verbindungslinien zu ziehen, eine von Th Mayer-Maly vor Langem aufgestellte Prämisse: ${ }^{21}$ Es kann für vergleichbare Falllagen nur eine begrenzte Zahl an rechtlich sinnvollen Lösungen geben, und es hat daher Ähnlichkeiten und Gleichheiten in den verschiedenen Rechtsordnungen gegeben, ohne daß sich daraus ein Einfluß erweisen ließe. Ein fremder Einfluß muß also nachgewiesen werden, und dafür genügen Ähnlichkeiten und Gleichheiten durchaus nicht.

3 Auf das Kapitel I „Perspektiven“ näher einzugehen, scheint angesicht der vorstehenden Ausführungen entbehrlich zu sein. Eigentlich ist mit ihnen bereits alles Wesentliche gesagt. Bartas Anspruch, sein enzyklopädisches Anliegen, die Fülle seiner Exzerpte und die umfassende Anlage seines Werks drohen jedoch, gerade Außenstehende in eine falsche Richtung zu weisen, weil ihnen die Kenntnis der realen Hintergründe fehlt. Was Bartas Meinung ist, könnte angesichts des monumentalen Charakters seines Werkes durchaus $\mathrm{zu}$ einer über Zitieren weiterwirkende

wieder Mängel, beispielsweise werden Abdrucke mal notiert, mal nicht. - S 511-561 sind allein die Titel der Unterabschnitte in die Kopfzeile übernommen worden; dies verwirrt. Siehe 184 Anm 795 ist eingangs offenbar „Vinogradoff“ (zit bereits Anm 793) ausgefallen. Ungut ergeht es Blass: II $^{2}$ zitiert als „Blaas“, und im Literaturverzeichnis auf 19793 statt auf 1979 datiert.

21 Th Mayer-Maly „Die Wiederkehr von Rechtsfiguren“ in Juristenzeitung (1971) S 1-3. 
antikrechtliche Darstellung werden. Das freilich ist weder Bartas Anliegen noch bei allem Respekt vor seiner Arbeit - seinen Ergebnissen angemessen.

„Kapitel I ist bestrebt - die Ausführungen in der Einleitung fortsetzend und vertiefend - auf weitere Grundlagen einzugehen" (S 57). Dieser einleitende Satz beschreibt den Inhalt von Kapitel I bestens. Es begegnet eine Fülle an Namen von Rechtshistorikern, Zitaten, Exzerpten und ausführlichen Textübernahmen. Jeder Gedanke, der eine Brücke vom griechischen zum römischen Recht auszudrücken oder auch nur anzudeuten scheint, wird in extenso vorgetragen; die Zeitgebundenheit vieler Auffassungen der Sekundärliteratur wird nicht hinterfragt. Viel wird dabei vor Augen geführt, was zur griechischen Rechtsgeschichte wenig bis nichts beiträgt. Ein Beispiel ist der kleine Unterabschnitt zur „Periodisierung der griechischen Rechtsentwicklung“ (S 75-77). Da werden verschiedene Zeitraster vorgestellt, ohne daß Barta Rechtsgeschichtliches über einzelne Schlagwörter hinaus berührte. Wenn man aber schon die Zeit vor 800 v Chr erwähnt, so hätten auch die Linear B-Schrift und deren Zeugnisse erwähnt werden sollen. Dabei handelt es sich vorwiegend um Notizen im Rahmen der zentralisierten Güterverwaltung in frühgriechischer Sprache, die zwar keine Rechtsurkunden enthalten, die aber vielfältige Details zu Wirtschaft und Gesellschaft erkennen lassen und folglich durchaus nicht völlig rechtsfern sind. ${ }^{22}$

Statt dessen konstruiert Barta „Einflußphasen des griechischen auf das römische Recht“ (S 77-84), die sich über rund 1000 Jahre hinweg und auf nahezu alle Gebiete der römischen Rechtsordnung erstreckt haben sollen. Wiederum wird mehr behauptet als bewiesen. Die von Barta als ,große und frühe griechische Gesetzgebungen“ bezeichneten Gesetze des Zaleukos und des Charondas hätten statt eines wenig aussagekräftigen Exzerpts aus einem 90 Jahre alten Werk von GM Calhoun ${ }^{23}$ und den Verweis auf Bartas spätere Erörterung doch wohl bereits hier einen wenigstens kursorischen Vermerk zu Historizität, Inhalt und Sekundärliteratur verdient. ${ }^{24}$ Barta übersieht übrigens durchaus nicht die Existenz und die Leistung der griechischen Kautelarjurisprudenz. Er thematisiert sie aber (bislang?) nicht eigens, sondern begreift sie als Teil seines oben bereits erwähnten, nahezu grenzenlosen Begriffs des griechischen Rechtsdenkens und mißt ihr ,nicht nur hervorragende praktische, sondern immer wieder auch theoretisch wichtige“ Bedeutung bei

22 Auf einen Nachweis der bislang nur vereinzelten Beiträge zum Thema wird hier verzichtet. Einen zweckgebundenen Überblick gibt beispielsweise BFr Steinmann Die Waffengräber der ägäischen Bronzezeit. Waffenbeigaben, soziale Selbstdarstellung und Adelsethos in der minoischmykenischen Kultur (Wiesbaden, 2012).

23 Siehe GM Calhoun „Greek law and modern jurisprudence“ in (1923) 9(5) California Law Review S 295-312 (302) [nv].

24 Vgl zur Gesetzgebung des Charondas und des Zaleukos K-J Hölkeskamp sv „Charondas“ in Der Neue Pauly Bd 2 (Stuttgart, 1996) Sp 1109/10; Ders Schiedsrichter, Gesetzgeber und Gesetzgebung im archaischen Griechenland (Stuttgart, 1999) S 130-144 uö (mwN) R Wolters sv „Zaleukos“ in Der Neue Pauly Bd 12/2 (Stuttgart, 2002) Sp 690. Siehe ferner M Mühl Die Gesetzgebung des Zaleukos und des Charondas (Leipzig, 1929); A Szegedy-Maszak „Legends of the Greek lawgivers“ (1978) 19 Greek, Roman and Byzantine Studies S 199-209. 
(vgl S 89). Subjektive Sichtweise und Empfinden charakterisieren auch den 2. Hauptabschnitt „Zum Wert humanistischer Bildung“ (S 93-122), der voll von Lesefrüchten ist, als Essay anregend zu lesen, aber ohne substantiellen Beitrag zur Fortwirkung des griechischen Rechts. Bezeichnend für eine wiederkehrende Unschärfe in der Argumentation ist der Unterabschnitt „Heinrich Mitteis und der Wert der Rechtsgeschichte“ (S 106-109). Lediglich die ersten Sätze reflektieren H Mitteis für den Stellenwert der Rechtsgeschichte wesentliche Studie „Vom Lebenswert der Rechtsgeschichte“. ${ }^{25}$ Der Rest besteht aus Assoziationen Bartas - Schwerlich zu billigen ist übrigens die mehrfache, unreflektierte, aber auch kritisierte Übernahme des Begriffs ,primitives Recht“. Es gibt kein ,,primitives Recht“. Jede Kulturordnung besitzt vielmehr die ihr gemäße Rechtsordnung, und die jeweilige Kulturordnung als ,primitiv“ zu bezeichnen entspringt einem kulturellen Überlegenheitsgefühl und spiegelt folglich Chauvinismus.

Nicht wesentlich weiter führt auch der folgende Abschnitt 3 ,Europa und griechisches Recht'“" (S 122-129). Wiederum findet sich eine Fülle an gedanklichen Zuordnungen zum ,griechischen Rechtsdenken“, ohne daß über die Behauptungen hinaus sachlich belastbare Ausführungen gemacht würden. Bezeichnend ist beispielsweise der Vorwurf, die römische Klassik habe nicht danach gefragt, „ob ein Rechtsinstitut nicht auch anders sinnvoll und gerecht gestaltet werden, oder ob es eine solche Gestaltung verdiente und der Korrektur bedurft hätte. $\left[{ }^{26}\right]$ Ganz anders der Zugang bei Platon, Aristoteles und Theophrast und überhaupt im griechischen Rechtsdenken, zu dem wesentlich sowohl die Rhetoren und Logographen als auch die Kautelarjurisprudenz beigetragen hat (sic!). Ihr Rechtsdenken beachtetet den gesellschaftlich-politischen und den wirtschaftlichen Zusammenhang des Rechts“" (S 124). Wohl wahr, und an dieser Stelle hätte Barta zwei einschlägige und ihm durchaus bekannte Arbeiten von E Klingenberg anführen können, welche bezeugen, daß den griechischen Philosophen das Recht des Alltags keineswegs fremd gewesen ist. ${ }^{27}$ Das gilt auch für andere Aspekte. So berichtet Diogenes Laertios, Aristoteles habe bei seiner Arbeit an der Athenaion Politeia 158 Verfassungen griechischer poleis herangezogen. ${ }^{28}$ Den Bezug zwischen Philosophie und Rechtsdenken haben J Triantaphyllopoulos und E Wolf umfassend aufgezeigt. ${ }^{29}$ Das ist letztlich

25 H Mitteis Vom Lebenswert der Rechtsgeschichte (Weimar, 1947).

26 Das muß man nicht so sehen, siehe beispielsweise die Beiträge in J-J Aubert / B Sirks (eds) Speculum Iuris. Roman Law as a Reflection of Social and Economic Life in Antiquity (Ann Arbor, 2002) (vgl dazu, allerdings höchst kritisch, J Hengstl in (2006) 88 Klio S 287-289).

27 Siehe E Klingenberg Platons NOMOI ГЕ $\Omega$ PГIKOI und das positive griechische Recht (Berlin, 1976); Dens „Bemerkungen zum platonischen Bienenrecht“ in Symposion 1971. Vorträge zur griechischen und hellenistischen Rechtsgeschichte, in Gemeinschaft mit J Modrzejewski und D Nörr hrsg von HJ Wolff (Köln / Wien, 1973), S 347-354 (vgl das vorstehend angeführte Werk S 40-49).

28 S Diog Laert, Vita Arist 27.

29 J Triantaphyllopoulos Das Rechtsdenken der Griechen (München, 1985); E Wolf Griechisches Rechtsdenken 4 in 6 Bdn (Frankfurt/M, 1950-1970) (beides bei Barta verzeichnet). 
nicht anders als der oben bereits erwähnte Bezug zwischen Dichtung und Recht. Den umgekehrten Nachweis, daß Philosophen, Rhetoren und Logographen die Rechtspraxis beeinflußt haben, bleibt B. zumindest einstweilen schuldig. Das ist auch nur schwer vorstellbar: Urkundenschreiber - Kautelarjuristen - hatten weder Zeit noch Anlaß, sich in theoretische Schriften zu vertiefen. Es gilt für den Alltag heutiger Rechtspraktiker nicht minder.

Die Abschnitte, „4. Die Phasen der römischen Rechtsentwicklung“ (S 129139), ,5. 'Andersheit' der griechischen Rechskultur“" (S. 139-158) und ,6. Gab es ein 'gemeines' griechisches Recht?" (S 159-215) bringen noch einmal eher theoretische Reflexionen, während „8. Rechtskollisionen im Archaischen Griechenland“ (S 345441); „9. Anfänge des Völkerrechts“ (S 442-511) dem geübten Recht gewidmet sind.

„7. Olympische Religion und Heroenkulte“ (S 215-344) ist ein Griff auf philosophisch-theologische Aspekte und deren Verbindung mit dem Recht. Dem einleitenden und auch weiter tragenden Gesichtspunkt, Recht lasse sich von den gesellschaftlichen Rahmenbedingungen (,religiöse Welt; Wert- und Moralmaßstäbe; Welt- und Menschenbild“, S 218) nicht abschichten, kann man nicht widersprechen. Allerdings: Wer Gras betrachtet, muß der ständig repetieren, wie weit die Sonne entfernt ist, die dieses Gras beleuchtet? Allein enzyklopädische Darstellungen erfordern eine derart umfassende Betrachtungsweise, und dazu gehört eine alltägliche Rechtsordnung durchaus nicht. Wieder einmal erstaunt hingegen Bartas Rückgriff auf ältere Literatur zu einer Problemstellung, zu der es an neueren Ausführungen durchaus nicht fehlt. Zur Entwicklung des Tötungsrechts und dem Verdrängen der Blutrache muß sich Barta zwar später noch eingehend äußern, die vielfach als diesbezüglicher Schlüsselaspekt erörterte Schildszene Il. 18.478-508 hätte Barta an dieser Stelle aber nicht stillschweigend übergehen dürfen. ${ }^{30} \mathrm{Zu}$ blaß bleiben die Bemerkungen zum delphischen Sakralrecht (S 224/5). Im übrigen zeigt gerade dieser religions- und rechtshistorisch geprägte Abschnitt die Problematik von Bartas Vorgehensweise. An handfesten Belegen mangelt es nämlich, und Barta muß sich daher weitgehend darauf beschränken, Meinungen zu referieren und zu addieren. In aller Regel handelt es sich um „Einzelstücke“. An einander gereiht ergeben sie durchaus ein plastisches Bild, aber noch lange keinen Beweis. Der läßt sich naturgemäß für die Frühzeit schwerlich erbringen. Allerdings ist zu fragen, ob wirklich alles einen überirdischen Kontext haben muß: Sollte den Menschen der Frühzeit wirklich entgangen und erst „dank göttlicher Hilfe“ bewußt geworden sein, daß Räuchern Fleisch konserviert? (S 231). Die Beispiele ließen sich mehren und auch auf andere Komplexe erstrecken. Der gesamte Abschnitt besteht aus kulturhistorisch interessanten Beobachtungen

30 Grundlegend HJ Wolff „Der Ursprung des gerichtlichen Rechtsstreits bei den Griechen“ in Beiträge zur Rechtsgeschichte Altgriechenlands und des hellenistisch-römischen Ägypten (Weimar, 1961) S 1-90; ferner ua E Ruschenbusch „Der Ursprung des gerichtlichen Rechtsstreits bei den Griechen" in Symposion 1977 (Köln-Wien, 1982) S 1-7 (= Ders Kleine Schriften zur griechischen Rechtsgeschichte (Wiesbaden, 2005) S 135-140). 
und Exzerpten. Sie tragen über das Kulturhistorische hinaus zur Rechtsentwicklung aber wenig bis nichts Substantielles bei. Zudem finden sich auch in diesem Abschnitt Mancherlei nicht Ausgeführtes, nur Erwähntes oder nicht weiter Belegtes. Der (philosophische) „Derveni-Papyrus“ ist als in Griechenland gefundener Papyrus selbst außerhalb der papyrologischen Welt sehr wohl bekannt, aber ein Eingehen auf die Fund- und Publikationsgeschichte im vorliegenden Rahmen zweifellos entbehrlich. Wenn der Papyrus jedoch (überflüssigerweise) schon erwähnt wird, sollte wenigstens eine Fundstelle angegeben werden. ${ }^{31}$ An gleicher Stelle (S 279) führt die Bemerkung zu ,frauenfeindlichen Werten“ nicht weiter. Es trifft zu, daß die Stellung der Frau in den verschiedenen Kulturen unterschiedlich gewesen ist. Das ist mit einem Hinweis auf das pharaonische Ägypten freilich keineswegs erledigt. Barta zeigt hierfür wenigstens an dieser Stelle weder Einzelheiten noch Gründe auf. Dabei wäre Vieles zumindest in aller Kürze erwähnenswert gewesen. Jeder Meister vor 100-150 Jahren, bei dem die Gesellen am Tisch der „Meisterin“ verköstigt worden sind, hätte da eine andere Meinung gehabt, und kein Bauer der Moderne kann auf die kluge Mitwirkung der Bäuerin verzichten. Dem entsprechend sagt ein Sprichwort „Was der Mann in Leiterwagen in die Scheuer fährt, trägt die Frau in der Schürze hinaus". Sollte das früher wirklich anders gewesen sein? Außerhalb der jeweiligen Oberschicht konnte auf die Mitwirkung der Ehefrau nur schwerlich verzichtet werden und das gilt nicht nur für das pharaonische Ägypten. ${ }^{32}$ Die Ehefrau ist beispielsweise ein unabdingbarer Wirtschaftsfaktor im Erwerbsleben der altassyrischen Kaufleute gewesen. Sie hat nämlich das Haus mitsamt den Geschäftsurkunden gehütet, während der Mann auf Geschäftsreisen unterwegs gewesen ist. ${ }^{33}$ Auch im klassischen Athen haben die Frauen nicht nur hinter dem Herd verborgen gelebt, ${ }^{34}$ und Frauen der römischen Oberschicht fehlte es durchaus nicht an wirtschaftlichem Spielraum. ${ }^{35}$ Die Beispiele ließen sich unschwer mehren. Nicht zuletzt in diesem Abschnitt macht B. erneut deutlich, daß und wie sehr es ihm um Rechtsdenken, nicht um Rechtsleben und Rechtspraxis geht. Er mischt all das freilich in Eins. Das Schwergewicht seiner Ausführungen bleibt aber das Rechtsdenken. Sein letztlich unentschiedene Vorgehensweise erlaubt freilich nur, nicht ohne Mühen sein unausgesprochenes

31 Erwähnt S 279. Siehe vor allem The Derveni Papyrus (ed with introd and comm by Th Kouremenos, GM Parássoglou \& K Tsantsanoglou (Firenze, 2006)).

32 Siehe S 279 unter Hinweis auf S Allam. Zum papyrologisch belegten Ägypten siehe beispielsweise Emanzipation am Nil Frauenleben und Frauenrecht in den Papyri hrsgg von $\mathrm{H}$ Froschauer \& $\mathrm{H}$ Harrauer (Wien, 2005).

33 Siehe J Hengstl „Zur Stellung der Frau nach den altassyrischen Urkunden“ in Antike Lebenswelten. Konstanz - Wandel - Wirkungsmacht. Festschrift für Ingomar Weiler zum 70. Geburtstag, hrsgg von P Mauritsch ua (Wiesbaden, 2008) S 243-263.

34 Siehe Chr Schnurr-Redford Frauen im klassischen Athen. Sozialer Raum und reale Bewegungsfreiheit (Berlin, 1996) passim. Siehe ferner zB E Ruschenbusch Untersuchungen zur Geschichte des athenischen Strafrechts (Köln / Graz, 1968) (abgedruckt in E Ruschenbusch Kleine Schriften [so Anm 30, S 75-123]).

35 SK Ermete Terentia und Tullia - Frauen der senatorischen Oberschicht (Frankfurt/M, 2003). 
Hauptanliegen zu verfolgen. Die Art seiner Gliederung erleichtert das keineswegs. Eben den Abschnitt 7 scheint nämlich eine „Zusammenfassung“ zu beschließen, deren Anfang zwar feststeht (S 297), nicht aber deren Ende. Ist es, wie man eigentlich vermuten muß, das Abschnittsende, das dann freilich erst 48 Seiten später kommt? Wie hat man die unbezifferten Unterabschnitte dieser Sequenz gedanklich einzuordnen?

Die dem Abschnitt 7 vorausgehenden Abschnitte 4-6 führen kaum weiter. Abschnitt 4, „Die Phasen der römischen Rechtsentwicklung“ (S 129-139) hebt zum einen auf das römische Fremdenrecht ab, zum anderen auf den Konservativismus der römischen Juristen, der sich mit dem römischen Fremdenrecht und erst recht mit griechischen Rechtsinstitutionen nicht leicht verbinden läßt. Was hat die (griechische) Proxenie, ${ }^{36}$ so läßt sich beispielsweise fragen, denn mit dem römischen „Fremdenrecht" zu tun? Unschärfen ergeben sich da nahezu zwangsläufig; beispielsweise kennt das attische Recht keine „Einreden“. ${ }^{37}$ „Platon und andere Philosophen arbeiteten nicht nur als Philosophen, sondern auch juristisch“ - wie denn, hatten sie Anwaltskanzleien oder betrieben sie Repetitorien? So einfach ist der Nachweis juristischer Tätigkeit nicht zu machen. ${ }^{38}$ Vieles bleibt dabei auf der Strecke, und das kann hier nicht nachgetragen werden. Ein Beispiel ist der Verzicht auf jeglichen Nachweis zu den artes liberales. ${ }^{39}$ B.unterscheidet vor allem nicht zwischen historisch Verbürgtem und aus unterschiedlichen Gründen tradiertem Erzählgut. Beides hat seine Berechtigung oder Erklärung, besitzt aber einen höchst unterschiedlichen Quellenwert.

Die Abschnitte ,5. 'Andersheit' der griechischen Rechtskultur?“ (S 139-158) und „6. Gab es ein 'gemeines' griechisches Recht?“ (S 159-215) bringen noch einmal eher theoretische Reflexionen. Vor allem setzt sich Barta mit F Pringsheim auseinander. ME schafft Barta hier eine Art Feindbild, welches er dann mit großem Aufwand ausbaut. In der Tat stellt Pringsheim fest, es habe im alten Griechenland keine privatrechtliche Literatur gegeben. ${ }^{40}$ Wem hätte sie denn genützt? Das Rechtsdenken der Philosophen ${ }^{41}$ haben die Urkundenschreiber garnicht erst zur Kenntnis genommen;

36 Siehe S 132. Zur Institution siehe ua C Marek Die Proxenie (Frankfurt/M, 1984) passim - bei Barta aufgeführt, nicht aber S 132 Anm 517.

37 So aber Barta S 134 ohne weitere Ausführung.

38 Im Sommersemester 2012 habe ich eine Lehrveranstaltung zum Thema „Waren sie Juristen? $\mathrm{Zu}$ den attischen Gerichtsrednern" durchgeführt, bei der ein Rechtsanwalt während einer Doppelstunde seine Tätigkeit geschildert hat. Vier Studierende erlangten den Grundlagenschein für das 1. Juristische Staatsexamen anhand von Referaten mit Noten von ,,befriedigend“ bis ,,sehr gut" und dem Ergebnis „Sie waren keine Juristen“. Mein Dank bzw meine Anerkennung gelten Herrn RA Zimmermann (Marburg) sowie stud iur L Bappert, L Boecking, A Dursun \& S Otte.

39 SK Visky Geistige Arbeit und die ,artes liberales“ in den Quellen des römischen Rechts (Budapest, 1977); ferner J Christes Bildung und Gesellschaft. Die Einschätzung der Bildung und ihrer Vermittler in der griechisch-römischen Antike (Darmstadt, 1975).

40 Siehe Barta S 142 sowie F Pringsheim The Greek Law of Sale (Weimar, 1950) S 2-5.

41 Vgl vor allem E Klingenberg aaO (o Anm 27). 
sie hatten mit dem Recht des Alltags genügend zu tun, und das betraf erstrangig die Beziehungen zwischen Privatpersonen. Ein „Gleichsetzen von Privatrecht und 'Rechtswissenschaft'“ (S 143) läßt sich darin nicht sehen - wahr ist freilich, daß die alltägliche Rechtsordnung vor allem durch Rechtsakte unter Privatpersonen repräsentiert worden ist. „Alle anderen Bereiche, also das gesamte öffentliche Recht, das Verfahrensrecht oder das Strafrecht wurden beiseite geschoben." (S 143). Das ist aus heutiger Sicht ein zutreffendes Urteil. Der Polisbürger jener Zeit hätte es wohl schwerlich verstanden und schon gar nicht zu teilen gewußt. Zudem ist diese Feststellung ebenso schwerwiegend wie ungenau. Wie definiert Barta die genannten Bereiche? Ist Strafrecht beispielsweise, was die Rechtsordnung Östereichs, Bartas Heimatland, dem Strafrecht zuordnet? Oder bestimmt die Sanktion, was Strafrecht ist, wie R Haase es für die altorientalischen Rechtssammlungen angenommen hat? ${ }^{42}$ Für das pharaonische Ägypten legt R Müller-Wollermann einen anderen Maßstab an, indem sie auf ,abweichendes Verhalten“ abstellt. ${ }^{43}$ Einen modernen Blickwinkel benutzt W Boochs bei seinen diversen Darstellungen zum pharaonischen Recht. Das macht Definitionen entbehrlich, kann aber nur ob der Anschaulichkeit willkommen sein. ${ }^{44}$ Auch zum athenischen wie zum römischen Recht sowie zu den gräkoägyptischen Verhältnissen liegen entsprechende Arbeiten sehr wohl vor. ${ }^{45}$ Die Beispiele ließen sich mehren, führen aber ohne sachgerecht entwickelte Grundlage nicht weit. So nämlich bezeugen sie höchstens eine alltägliche Gewaltbereitschaft und deren Bekämpfung durch die Staatsgewalt. Ein Grundkonzept, ein Anspruch des Staates auf alleinige Ausübung der ordnenden Gewalt in seinem Gebiet ist dem nicht zu entnehmen. „Strafrecht“ ist demnach inhaltlich nicht einfach vorauszusetzen, sondern bedarf der Definition.

42 Siehe R Haase „Körperliche Strafen in den altorientalischen Rechtssammlungen“ in (1963) 10 Revue Internationale des Droits de l'Antiquité S 55-75; ferner H Neumann „Zum Strafrecht in den ältesten Gesetzen Mesopotamiens“"(1989) 15 Das Altertum S 13-22; U Sick Die Tötung eines Menschen und ihre Ahndung in den keilschriftlichen Rechtssammlungen unter Berücksichtigung rechtsvergleichender Aspekte (Jur Diss, Tübingen, 1984).

43 R Müller-Wollermann Vergehen und Strafen: Zur Sanktionierung abweichenden Verhaltens im Alten Ägypten (Leiden / Boston, 2004).

44 W Boochs Die Finanzverwaltung im Altertum (St Augustin, 1985) (keineswegs nur zu Ägypten); Ders Strafrechtliche Aspekte im altägyptischen Recht (Sankt Augustin, 1993); Ders Altägyptisches Zivilrecht (St Augustin, 1999) (Überblick über Quellen, Grundsätze und Institutionen des „Zivil“"rechts der Pharaonenzeit samt den Grundzügen einer Urkundenlehre und des Gerichtsverfahrens).

45 Siehe E Ruschenbusch „Das Vergehen und dessen Ahndung nach griechischem Recht“ in (1988) 95 Gymnasium S 369-374 (abgedruckt in E Ruschenbusch Kleine Schriften [so Anm 30] S 188192); B Baldwin „Crime and criminals in Graeco-Roman Egypt“ in (1963) 42 Aegyptus: Revista italiana di Egittologia e di Papirologia S 256-263; W Kunkel Kleine Schriften. Zum römischen Strafverfahren und zur römischen Verfassungsgeschichte (Weimar, 1974). Th Mommsen's Alterswerk Römisches Strafrecht (Leipzig, 1899) ist noch immer von großem Wert, in der Sichtweise aber vielfach überholt. 
Entsprechendes gilt für die „Andersheit“ des griechischen Rechts wie der Frage, in wie weit man von einem „gemeingriechischen Recht" sprechen darf oder gar sprechen muß. Geht man nämlich von der Annahme aus, daß jede Kulturordnung das ihr angemessene Recht besitzt, so entbehrt der Gedanke an eine „Andersheit“ des griechischen Rechts, an einen ,griechischen Sonderweg“ von vorn herein der Überzeugungskraft. Irgendwie muß die griechische Rechtsordnung ja anders gewesen sein als andere Rechtsordnungen, sofern nicht die Gegebenheiten übereinstimmten und vergleichbare Rechtsfolgen hatten. „Andersheit" und "Sonderweg“ sowie „gemeingriechisches Recht“ sind die diesbezüglichen Begriffe, denen Barta folgerichtig nachgeht und seinen Ausführungen ist daher zu folgen. Dazu gehört die Frage, in wie weit es ein „griechisches Recht“ gegeben hat - „'gemeines' griechisches Recht" drückt Barta es bewußt altertümelnd aus. Kriterium kann nur sein, wie man „Gemeinsamkeit" versteht. Hier ist zweifellos Bartas Hinweis auf die moderne Rechtskreislehre angemessen (S 161). Der Gedanke sollte $\mathrm{mE}$ dennoch zurückhaltend verwendet werden, denn die antiken „Rechtskreise“ entsprechen nun einmal nicht den modernen Gegebenheiten. Barta sieht das und negiert es dann doch (S 162), offenbar weil er eine bestimmende Leitlinie annimmt (S 160: „Leitpoleis“). Wiederum taucht die Schimäre eines alles umfassenden Rechtsdenkens auf, obgleich Barta Abweichungen (in Maßen) in Rechnung stellt. ${ }^{46}$ Eine jede Rechtsordnung, insbesondere eine kulturell entwickelte, muß gesellschaftliche Werte, Familiengliederung, familiäre Vermögensbestimmungen, Erbfolgeregelungen und Anderes mehr kennen - Ähnlichkeiten und Gleichheiten können daher in diesem Bereich wie auch in Wirtschaft, Handel und sonst kein besonderes Merkmal sein.

Viele der von Barta in diesen Abschnitten vorgetragenen Beobachtungen sind unbelegt. ME macht Barta gerade hier die wesentliche, zweifache Schwäche seiner Betrachtungsweise deutlich. Er „verwirkt" zum einen im wahrsten Sinne des Wortes die rechtskulturellen Aspekte so, daß er im Wechselspiel der Argumentation mal den einen, mal den anderen anspielen kann. Zum anderen compiliert Barta die von ihm als einschlägig erachteten Meinungen der Sekundärliteratur derart, daß das von ihm angestrebte Bild entsteht. Dieses ist ein in vielfältiger Beziehung von griechischen Vorgaben abhängiges römisches Recht. Bartas Argumente können hier nicht im Einzelnen zusammengestellt und widerlegt werden. Einige Beispiele müssen genügen und sollen folgen.

Bezeichnend für Bartas anspielenden statt argumentierenden Vortrag ist beispielsweise (S 174): „Der Freundschaftsvertrag zwischen Ephesos und Sardes 'aus der Zeit des Prokonsulats des jüngeren Scaevola, Q. Mucius Scaevola P. Fil., aus dem Jahre 98 v. Chr.' enthielt internationales Strafrecht: forum rei, forum comprehensionis: 'Solche Vergünstigungen begegnen auch in anderen griechischen

46 Zum syrisch-römischen Rechtsbuch, zit Anm 682, ist der Hinweis auf E Weiss (1923) bibliographisch durch W Selb \& H Kaufhold Das syrisch-römische Rechtsbuch (Wien, 2002) zu ergänzen. 
Staatsverträgen und erinnern an die Fälle, in denen in Rom der Praetor die im Zivilrecht auf den Bürger zugeschnittene Klage ficta civitate den Peregrinen zugesteht.' [Anm 737: Gaius IV 37]“. Schreibt so Gaius, und ist jener Vertrag nach Inhalt und Fundstelle einsichtig? Polisgründungen in Ägypten gibt es nur drei bzw vier (Naukratis; Alexandria; Ptolemais, Antinoupolis); ${ }^{47}$ dennoch steht Milet als Mutterstadt für ,zahlreiche (etwa 80?) Tochterstädte im Schwarzmeergebiet und in Ägypten“ (S 174). Von der Rechtsordnung des im pharaonischen Ägypten gegründeten Naukratis ist zwar nahezu nichts bekannt - immerhin: „Eine Facette des kulturellen Hegemoniestrebens einzelner griechischer Poleis - etwa auch Athens bildete ihr Rechtssystem“ (S 175).

Der letzte Abschnitt, „10. Rezeption durch Rom?“"(S 511-561), ist offensichtlich die Quintessenz des ersten Bandes. Barta schreibt in diesem Abschnitt, für seine Auffassung und Methodik höchst bezeichnend „Weshalb haben gerade im Rechtsbereich immer wieder Transfers und Rezeptionen stattgefunden?"(S 516). Haben sie wirklich stattgefunden? Barta behauptet das immer wieder, bleibt stichhaltige Nachweise aber letztlich schuldig. Die Möglichkeit der Übernahme ersetzt nicht deren Beweis, und in so weit ist wie so oft in der antiken Rechtsgeschichte auf die von Th Mayer-Maly aufgestellte und oben bereits wiedergegebene Prämisse zu verweisen: Es kann für vergleichbare Falllagen nur eine begrenzte Zahl an rechtlich sinnvollen Lösungen geben. Barta übersieht darüber hinaus, daß eine ganze Reihe von Gegebenheiten, welche der rechtlichen Gestaltung bedurft haben, letztlich überall wenigstens in der Welt des antiken östlichen Mittelmeeres mehr oder minder vergleichbar vorgelegen haben. Das gilt für die Subsistenzwirtschaft, die den Alltag der meisten Menschen bestimmt hat. Es gilt aber auch für die überregionalen Gegebenheiten. Seit vorgeschichtlicher Zeit hat es umfangreiche Handelsbeziehungen gegeben, und die ließen sich keineswegs mit dem Schwert in der Hand durchführen. Selbst kriegerische Auseinandersetzungen bedurften letztlich gewisser Regularien, um zu einem zukunftsfähigen Ende zu gelangen. Die Notwendigkeit eines geregelten Miteinanders hat also in jedem Bereich zu allen Zeiten bestanden. All das bietet hinreichend Spielraum für die Erklärung, es könne für vergleichbare Falllagen nur eine begrenzte Zahl an rechtlich sinnvollen Lösungen geben. Gemeinsame Herkunft, Sprache und Religion begründen zweifellos gemeingriechische Übereinstimmungen, führen aber doch nicht zwingend zur Schaffung eines gemeinsamen Rechts. Die Betonung hier liegt auf „Schaffung“ (vgl S 179); die Existenz überkommener gemeingriechischer Rechtsinstitutionen soll damit keineswegs geleugnet werden. Das Gedankenspiel mit Einflüssen der über

47 Allgemein zum Recht der poleis im ptolemäischen Ägypten siehe vor allem J Modrzejewski „La règle de droit dans l'Égypte ptolemaïque (État des questions et perspectives de recherches)“ in Essays in Honor of C. Bradford Welles (New Haven, 1966) S 67-77, sowie Ders Loi et coutume dans l'Egypte grecque et romaine (Paris, 1970) S 92-113. Zu Naukratis siehe zB W Helck sv „Naukratis“ in Der Kleine Pauly Bd 4 (München, 1972) Sp 10. 
zweitausend Jahre umfassenden ägyptischen bzw. keilschriftlichen Rechtsordnungen ist im Übrigen weder fundiert noch überzeugend (zB S 189). Barta übersieht dabei immer wieder gänzlich, daß die von ihm erwähnten kulturellen Kontakte und von ihm referierten Meinungen der Sekundärliteratur eines nicht belegen, nämlich eine praxiswirksame, zeiten- und länderübergreifende „Rechtspolitik“ - die hat es eben nicht gegeben. Es fällt übrigens auf, daß Barta bei all' seinen diesbezüglichen Reflexionen fast nur auf Nichtjuristen zurückgreift. Bartas Fülle an Beobachtungen ist jedoch immer wieder beeindruckend. Im Ergebnis leidet sie aber an der weit weniger analytischen als assoziativen Vorgehensweise.

„8. Rechtskollisionen im Archaischen Griechenland“ (S 345-441) und „9. Anfänge des Völkerrechts“ (S 442-511) geben eher als die übrigen Abschnitte die tatsächlichen Gegebenheiten wieder. Exzerpieren, Referieren und Katalogisieren beherrschen allerdings auch diese Abschnitte. Quellenwiedergaben oder eigenständige Quellenexegesen sucht man vergeblich. Immerhin sind die Schilderungen der Kolonisation und der Beziehungen zwischen Metropolis und Apoikie sowie damit zusammenhängender Fragen anschaulich, tragen zur Rechtsordnung freilich nicht viel bei, vor allem kaum Substantiertes. So bleibt beispielsweise dunkel, inwiefern die Koloniegründungen ,schon die kautelarjuristische Phantasie in den Mutterstädten gefördert, ja beflügelt hatte“ (sic). Beachtenswerte Bemerkungen von Barta gehen unter diesen Umständen (nicht nur hier) unter. Die Verwendung modernrechtlich geprägter Terminologie hilft zudem kaum weiter. An anachronistischen Wertungen fehlt es in diesem Zusammenhang nicht. „Kollisionsrecht zwischen Mutter- und Tochterstadt ist eine Vorstufe des modernen 'nationalen Privatrechts'" (S 366). Eine derartige Rubrifizierung bringt rechts-ideengeschichtlich ebensowenig weiter wie Bartas Differenzierung in ,innerstaatliches“ beziehungsweise „intermunizipales“ „Kollisionsrecht“ (S 348). Daß municipium ein Begriff einer außergriechischen Rechtsordnung ist, soll hier nicht thematisiert werden. Wohl aber ist zu fragen, in wie weit derartige Kollisionen tatsächlich gefühlt geworden sind. Schwerlich im ptolemäischen Ägypten, denn da sind die Verträge mal griechisch, mal demotisch abgefaßt worden, bar jeder „Kollision“, denn die hat gewöhnlich bereits die Wahl der Sprache vermieden.Und wenn nicht, dann die Kunst der Urkundenschreiber. Das heißt nicht, daß es keine wechselseitigen Einflüsse gegeben haben mag. Nur sind diese erst zu entdecken, und bei Barta findet sich dazu nichts. Bartas Ausführungen unter dem Gesichtspunkt „Kollisionsrecht“ müssen nicht nur hier mit Bedacht betrachtet werden. Dies gilt nicht minder für Bartas Ausführungen in den Unterabschnitten „Das 'Internationale Privatrecht' der Antike“ (S 368-376) und „'Intermunizipales' Kollisionsrecht zwischen Mutter- und Tochterstadt" (ab S 377; das Abschnittsende ist nicht sicher zu bestimmen). Die denkbaren Möglichkeiten sind umfassend dargestellt. In welchem Maße es eines solchen „Kollisionsrechts“ überhaupt bedurft hat, wird jedoch nicht erörtert. Man stelle sich vor: Menschen einheitlichen Herkommens und politischer Überzeugung trennen sich räumlich aus ökonomischen Gründen. Wie tief 
wird dann auf absehbare Zeit die rechtliche Trennung sein? An unterschiedlichen Auffassungen und am Bedürfniss, diese beizulegen, wird es nicht gemangelt haben. Aber darf man deren Regelung wirklich als „Kollisionsrecht“ bezeichnen, während es im Privatrecht der „Dissenz“ ist? Daß es an „internationalen“ Konflikten nicht gemangelt hat, belegt wohl die Beiziehung auswärtiger Richter, die vielfach in Inschriften zu ihren Ehren belegt sind. ${ }^{48}$ Die - von Barta nicht erörterte - Rolle solcher externer Richter bedarf zweifellos noch eingehenderer Erhellung. Auch sonst benötigt in diesem Zusammhang noch Vielerlei der genauen Analyse. Eine wesentliche Frage kann nur sein, in wie weit das gelebte Recht all' dem entsprochen hat. Aus dem außerägyptischen Bereich lassen uns die Quellen dazu weitgehend im Stich. Besser ist es nur um die Gegebenheiten im ptolemäischen Ägypten bestellt. Aber um die geht es hier kaum.

Schwerlich folgen läßt sich Bartas apodiktischen Feststellungen „Kolonisation fördert Rechtsvereinheitlichung und autochthone Kautelarpraxis“" (S 353) oder die Koloniegründungen hätten „schon die kautelarjuristische Phantasie in den Mutterstätten gefördert, ja beflügelt“ (S 362). Die „Kautelarjuristen“, die Urkundenschreiber, werden an dem Geschehen gedanklich nur teilgenommen haben, sofern sie einen entsprechenden Vertrag aufzusetzen hatten. „Das 'Internationale Privatrecht' der Antike“ (S 368) ist gleichfalls eher Schlagwort als weiterführend. „Internationales Privatrecht“ bezeichnet im heutigen Recht die Gesamtheit der nationalen Rechtssätze, die bei Auslandsberührung bestimmen, welche von mehreren möglichen internationalen Privatrechtsordnungen anzuwenden ist. Für die griechisch-hellenistischen Gerichte ist das freilich ohne Belang gewesen, denn die Parteien hatten die rechtlichen Bestimmungen, auf die sie sich beriefen, als Beweise vorzulegen. Es blieb dann vor allem dem Geschick der Partei überlassen, was als Recht zur Anwendung kam.

Abschnitt 8 ist wiederum von einer anachronistischen Betrachtungsweise geprägt. Die Kolonisierung scheint - nach Barta - von amtlicher Planung bestimmt gewesen zu sein, und die Ausführungen zum Internationalen Privatrecht vernachlässigen völlig die Beweispflicht der Parteien. Diese Bemerkungen sollen hierzu denn genügen. Ein letzter Blick gilt noch Abschnitt 9 „Anfänge des Völkerrechts“. Er enthält eine Fülle an Informationen zum Thema seit den ältesten Zeiten. ${ }^{49}$

4 Es fällt schwer, ein Votum über Bartas Werk anhand des ersten Bandes abzugeben. Fachlich ist es leider leicht. Bartas ständiges Bemühen, jegliche selbst

48 Vgl dazu (ua) K Harter-Uibopuu Das zwischenstaatliche Schiedsverfahren im achäischen Koinon. Zur friedlichen Streitbeilegung nach den epigraphischen Quellen (Köln / Weimar / Wien, 1998); D Nörr ,"Richter aus der Fremde’ und römische Provinzialgerichtsbarkeit. Bemerkungen zu IG. XII 5.722 (IG. XII Suppl p 127)“ in (1998) 26 Index S 71-87; Ch V Crowther „Aus der Arbeit der 'Inscriptiones Graecae' IV. Koan Decrees for Foreign Judges“ in (1999) 29 Chiron S 251-319.

49 Bartas Ausführungen zu den „Kodifikationen sumerischer Stadtstaaten“ (S 458-461) sollte man nicht kritiklos übernehmen. 
nur mögliche griechische Spur über Zeit und Raum hinweg mit der römischen Welt zu verbinden, vermag in aller Regel nicht zu überzeugen. Barta unterscheidet zudem nicht zwischen Rechtsdenken, Rechtsanwendung durch Logographen und Praktikern des Alltags. Entsprechendes gilt für die Durchführung. Barta zitiert und paraphrasiert in einem höchst ungewöhnlichen Maße, verzichtet aber darauf, seine Lesefrüchte inhaltlich zu analysieren. Er stellt sie mit verbindenden Worten nebeneinander und geht offenkundig davon aus, daß sie auf diese Weise überzeugen. Gedanklich vergleichbar sind seine ständig wiederkehrenden Vorwürfe, selbst Koryphäen der Altertumswissenschaften hätten ihm, Barta, wichtig dünkende Aspekte übergangen. Barta übersieht dabei, daß es in der wissenschaftlichen Diskussion eben nicht um „Rundumschläge“, sondern um Konzentration auf das Wesentliche geht. Dazu gehört auch, daß eine sich ständig mehrende Fülle an neuen Zeugnissen und Erkenntnissen aufgearbeitet werden muß, ehe man sich an neue Synthesen machen kann. Seine Belesenheit und seinen Gedankenreichtum möchte man freilich ungern missen. Bartas Werk liest sich, da es keine tragende Linie außer dem im Titel ausgedrückten Gedanken gibt, wie eine Fülle an Essays - so sollten sie vielleicht auch künftig veröffentlicht werden! Und Mancherlei hat Barta dem entsprechend anderwärts publiziert.

5 Der zweite Band ist ob seines Umfangs zweigeteilt; er enthält Kapitel II von Bartas umfangreichem Werk. Barta bezeichnet Kapitel II im Titel mit „Archaische Grundlagen“ und hat es im Text mit „Drakon, Solon und die Folgen“ bezeichnet. ${ }^{50}$

Der Titel „Archaischen Grundlagen” verwirrt zunächst, denn mit „Archaischen Grundlagen" verbindet nicht jeder Leser die Gesetzgeber der beginnenden (athenischen) polis Solon und Drakon, sondern eher die Zeit der Schriftgewinnung (8. Jh v Chr), der vorausgehenden, sich zunehmend erhellenden „dunklen“ Epoche und der Linear-B-Zeit. In der Anlage entsprechen die beiden Teile dem ersten Band, enthalten also insbesondere Glossar, Literaturverzeichnis und Stichwörter; das Quellenverzeichnis hingegen ist neu und willkommen. Nicht recht befriedigen will, daß die beiden Teilbände völlig getrennt angelegt sind, einschließlich der Paginierung. Da hätte eine Aufteilung in zwei selbständige Bände der Übersicht und der Handhabbarkeit wohl eher gedient, zumal Barta sich ohnedies genötigt gesehen hat, den letzten Abschnitt, Abschnitt 23 „Rezeption und Kulturtransfer aus dem Alten Orient", in Band III zu verweisen, um das Erscheinen von Band II nicht zu

50 Band III soll mit den Kapiteln III-VI („Die 'Eumeniden' des Aischylos“; „,Der ‘Melierdialog’ des Thukydides“; ,Euripides und das Naturrecht“ sowie „Gab es eine griechische Jurisprudenz“) den zweiten Teil („Recht, Dichtung und Geschichte“) sowie das erste Kapitel des dritten Teils („Praxis und Theorie griechischen Rechtsdenkens") enthalten; der vierte Band die restlichen drei Kapitel des dritten Teils („Gab es eine griechische Jurisprudenz?“; „Platon“; „Aristoteles und Recht“) sowie die Teile vier und fünf (Recht, Religion und Gerechtigkeit“; „Ausblick und Ergebnisse“); die Kapitel IX („Recht und Religion“) beziehungsweise X („Epilog“; „Zusammenfassung und Thesen") (siehe die detaillierte Inhaltsübersicht S XIII/XIX). 
verzögern $\left(\mathrm{BII}^{2}, \mathrm{~S} 375\right)$. Die übrige Gestaltung entspricht ebenfalls dem ersten Band: eine Vielzahl an Exzerpten und Literaturreferaten sowie umfangreiche Ausführungen in zahlreichen Fußnoten. Auch der zweite Band zeugt von Bartas Belesenheit, seinem enzyklopädischen Wissen und seinem Bemühen, vor allem anhand der in der Sekundärliteratur vertretenen Meinungen assoziativ Verbindungen über das griechische Recht hinaus zu ziehen. Die Quellenlage ist dabei mitunter kärglich, und Barta $m u ß$ daher vielfach mit Hypothesen arbeiten, deren hypothetischer Charakter freilich oftmals nicht ohne Weiteres zu erkennen ist. Allerdings spiegeln sie zweifellos Bartas Überzeugung.

Vor der inhaltlichen Vorstellung des Bandes II soll zunächst der Blick auf Bartas Sichtweise, Aufbau und Vorgehensweise vertieft werden. Sie entsprechen Band I: B. sammelt Befunde, Lesefrüchte und Exzerpte aus der Sekundärliteratur und verbindet sie derart, daß das Bild einer von der Antike, sogar der vorgriechischen Zeit, bis heute reichenden, zumindest bis heute fortwirkenden Entwicklung entsteht. Bd II $^{1}$ umfaßt 10 Abschnitte, Bd. II $^{2}$ die Abschnitte 11-22. Es liegt auf der Hand, daß diese Abschnitte weder dem Umfang noch dem Inhalt nach in vertretbarer Zeit von einem Einzelnen detailliert zu würdigen sind. Der Band wird daher im Folgendenden lediglich vorgestellt, und dabei oder im Anschluß daran werden einzelne Schwerpunkte näher gewürdigt. Barta betont die mit seinem Thema gerade in diesem Band verbundenen Schwierigkeiten in seiner Einleitung (S 1-14) mit Recht. Zugleich unterstreicht diese Einleitung wiederum Bartas Vorgehensweise in positiver wie in negativer Weise.

Revidiert man die beiden ersten Bände, so fällt immer wieder auf, in welch' starkem Maße Barta bemüht ist, Verbindungslinien über das griechische Recht hinaus herauszuarbeiten, so als bedürfe die griechische Rechtsordnung einer Rechtfertigung - einer Rechtfertigung, die vor allem Bartas Werk liefern soll, indem Barta einer Fortwirkung des griechischen Rechts das Wort redet. Ein wichtiges Moment ist, daß Barta - wie bereits erwähnt - alle denkbaren Aspekte in Eins gießt und so nahezu unbeschränkten Manövrierraum hat. Alle denkbaren Aspekte von Was ist zu fragen, und die Antwort kann nur lauten: von Allem, was sich nur irgendwie mit dem Begriff „Recht“ in Verbindung bringen läßt. Beispiele drängen sich bereits ab den ersten Seiten immer wieder auf. So beginnt der Persönlichkeitsschutz mit Solon, setzt sich über Kleisthenes und das perikleische Athen fort, um von dort nach Alexandria zu dringen und danach das römische Rechtsdenken zu beeinflussen (BII $\left.{ }^{1}, \mathrm{~S} 3\right)$. Das Gedankenspiel ist reizvoll und theoretisch sogar möglich. Fakten, es zu untermauern, sind jedoch nicht ersichtlich.

Barta scheint freilich weniger bloß Verbindungslinien aufzeigen zu wollen, sondern immer wieder den Fortbestand und das Weiterwirken eines einmal gefaßten und von ihm erkannten Rechtsgedankens. An Beispielen ist kein Mangel, und Barta unterstreicht sie ständig mit Hinweisen auf das moderne, meist österreichische Recht ${ }^{51}$ 
- teilweise auch als Desiderat. ${ }^{52}$ Ein Beispiel sind seine Ausführungen zur غ̇ $\pi \varepsilon \varepsilon i ́ \kappa \varepsilon ı \alpha$ „Billigkeit (o.ä.)“ (BII ${ }^{2}$ 13). Barta betrachtet diesen außerhalb der Primäliteratur offenbar nicht besonders oft verwendeten Begriff $\mathrm{f}^{53}$ als weiterwirkendes Urbild eines umfassenden Korrektivs fester Regeln der Rechtsordnung. Entsprechendes kann man zu Bartas Ausführungen zur Hybris erkennen, die im Persönlichkeitsschutz münden (BII $\left.{ }^{2} 146\right)$.

Der zweite Band bestätigt ferner eine Sichtweise auf die Sekundärliteratur, die einmal mehr Bartas Belesenheit bezeugt, nicht aber irgendein Verständnis dafür, daß Wissenschaftler wie Literaten in ihrer Zeit wie in ihrer Kenntnis zeitund sachgebunden schreiben. Die von Barta gewünschte Gesamtschau läßt sich nicht einmal in einem derart monumentalen Werk erreichen, wie Barta es bietet beziehungsweise mit den Folgebänden anstrebt. Immer wieder bemängelt Barta die Äußerungen anderer, vorausgehender Vertreter der Antiken Rechtsgeschichte, da diese irgendwelche von Barta als wichtig erachtete Aspekte seiner Meinung nach nicht hinreichend berücksichtigt haben. Bartas Methode, Exzerpte aus der Sekundärliteratur mit verbindenden Worten aneinander zu reihen, um so seine Anschauungen zu entwickeln und zu belegen, ist zudem alles Andere als klar und überzeugend. Um die wiedergegebenen Stellen kritisch zu bewerten, müßte der originale Zusammenhang eingesehen werden. Das wäre zu vermeiden gewesen, wenn Barta die Gedanken der Vorlage in seine Erwägungen eingearbeitet und auf Primär- und Sekundärbelege lediglich verwiesen hätte. Es hätte zudem den Umfang von Bartas Darlegungen stark verringert.

Bartas Umgang mit Primärquellen dürfte gleichfalls in seiner Vorgehensweise begründet sein. Barta zitiert und analysiert bislang keinen Quellentext in extenso, sondern bringt Meinungen und Analysen der Sekundärliteratur, gewöhnlich ohne exakte Textbezeichnungen anzugeben. Das ist mitunter bloß frustrierend, etwa wenn „das Beispiel aus dem Fayum im ptolemäischen Ägypten“ (Bd I, S 348) über die Anm 1677 auf die Anm 1786 und Meinungen von H Lewald und E Schönbauer verweist, bar eines jeden Beleges. Gravierender ist Bartas Darstellung der Inschrift SEG XXXIII 690 (S 22). Barta gibt den Kommentar einer Inschriftensammlung wieder. Die Inschrift ist inzwischen allerdings textlich erweitert und erneut publiziert

und zwar des Menschenrechts- und Grundrechtsschutzes, wie des straf- und privatrechtlichen (durch ein allgemeines Persönlichkeitsrecht; siehe die §§ 16 und 17 AGBGB[,] die diesem Vorbild am nächsten kommen und noch mehr zu leisten imstande wären als bisher) ${ }^{\mathrm{Anm}} 1065$ : „So könnte aus $\S 17$ AGBGB die fehlende Popular-Klagslegitimation in Fällen wie jenem der mißbräuchlichen Verwendung des Namens ‘Einstein’ abgeleitet werden; siehe mein Zivilrecht 2004, I 258. - ...“.

52 Siehe beispielsweise zum „Allgemeinen Persönlichkeitsrecht“: „Selbst die dem Wortlaut nach vorhandene Anerkennung durch $\S 16$ [österreichisches] AGBG (das dtBGB kennt einen solchen Schutz bis heute nicht), wurde erst in den späten 1960er und den 1970er Jahren von der Rechtsprechung fruchtbar gemacht und österreichische Rechtsdogmatiker haben sich bis zur jüngsten Jahrtausendwende gegen eine solche Annahme gewehrt“ (BII² S 149 Anm 913).

53 Vgl zB A Greek-English Lexikon hrsgg von HG Liddel \& R Scott (Oxford, 1996) S 632; Fr Preisigke Wörterbuch der griechischen Papyrusurkunden (Heidelberg, 1922 ff) Bd 1 Sp 552/3. 
und auf die 2. Hälfte des 5. Jahrh v Chr herunterdatiert worden. ${ }^{54}$ Des Weiteren sind fehlende Textbezeichungen wie „Graeca Halensis und Dikaiomata-Papyrus“ (S 3) oder „Fayumtexte“ (Bd I, S 436) schlicht unbrauchbar. Im ersten Fall handelt es sich um $P$ Hal I 1; „Graeca Halensis“ bezeichnet die Herausgeber, ${ }^{55}$ und dikaioma „1) Rechtsanspruch; Rechtstitel; 2) Beweisurkunde“ ist ein Terminus, welcher keineswegs allein in P Hal I 1, 38 erscheint. ${ }^{56}$ Die Großoase Fayum, der antike nomos Arsinoites, ist das Herkunftsgebiet einer Unzahl griechischer Papyri aller papyrologisch einschlägigen Entstehungszeiten. Ein unspezifischer Hinweis auf von dort stammende Texte ist daher belanglos. Völlig überflüssig ist ferner die (beleglose) Schilderung der Entnahme von Papyrus-Füllmaterial aus Krokodilmumien (Bd 1, S 375), und Bemerkungen zur Verwendungen von Mumienkartonnage hätten zum Thema ebenso wenig beigetragen, denn gewöhnlich kommt es nicht auf die Fundsituation, sondern auf den Inhalt der Quellen an.

Bartas Umgang mit den Primärquellen ist nicht minder befremdlich. Barta zitiert sie gewöhnlich nur anhand von Sekundärliteratur, ohne den griechischen Text wiederzugeben und vielfach ohne aktuelle Textbezeichnung. Eine nachvollziehende Textkritik oder Bewertung von Bartas Ausführungen ist daher nicht möglich, sofern man sich nicht schlicht an den wiedergegebenen Wortlaut halten will.

6 Auf den Inhalt von Band II = Kapitel 2 soll, wie bereits angekündigt, hier nur kürzer eingegangen werden. Die inhaltliche Darstellung des zweiten Bandes ist wie bei Band I nicht leicht dank der zahlreichen Verweise auf die Folgebände wie auf das Vorausgehende. Barta knüpft insgesamt an Person und Werk von Drakon und Solon, im Wesentlichen an Letzteren.

Im ersten Abschnitt (,Von Solon bis Kleisthenes“; BII ${ }^{1}$ S 13/15-58) ordnet Barta zunächst Solon in den Kreis der Sieben Weisen ein, sammelt Biographisches und schildert Solons Bedeutung und Wirken. ${ }^{57}$ Dabei geht er auch

54 Siehe MN Tod (ed) A Selection of Greek Historical Inscriptions (Oxford, 1933) Nr 1, Kommentar aaO S 2. Zum Text siehe nunmehr JH Oliver, Text of the so-called Constitution of Chios from the first half of the sixth century B.C." in (1959) 80 The American J of Philology S 296-301.

55 Dikaiomata: Auszüge aus alexandrinischen Gesetzen und Verordnungen in einem Papyrus des Philologischen Seminars der Universität Halle mit einem Anhang weiterer Papyri derselben Sammlung Graeca Halensis (ed) (Berlin, 1913). In Bd II² S 174-181 paraphrasiert Barta allerdings P Hal I 1 unter dem Gesichtspunkt, der Papyrus enthielte eine „Generalklausel für Hybris“. Das, wie bei Barta üblich, nur in Übersetzung wiedergegebene Exzerpt (BII ${ }^{2}$ S 176-177) betrifft freilich nicht die Hybris, sondern ist wohl beispielshalber gemeint. Bartas Ausführungen zur Hybris in P Hal I 1 (BII² S 178-181) sind auf eine „Entwicklung eines allgemeinen Persönlichkeitsrechts und eines Schutzes der Menschenwürde im griechischen Rechtskreis“ gerichtet.

56 Vgl (ua) Fr Preisigke (so Anm 53), Sp 382/3. Bezeichnend ist, daß HJ Wolff Das Justizwesen der Ptolemäer (München, ${ }^{2} 1970$ ) den Begriff nicht verzeichnet.

57 Vgl zu diesem Abschnitt DF Leão Sólon. Ética e Política (Lisboa, 2001) der in seinem ersten Hauptteil (S 19-212) ua auf die Wirkungsgeschichte von Attitographen und Logographen eingeht, aber auch unter „A formação do conceito de Patrios Politeia“ (S 43-72) zu den politischen, 
auf „Frühe Polissatzungen“ ein, ferner auf „Solons staatsrechtliche Reformen“. Den Unterabschnitt „Entstehung des Rechtssubjekts“ (BII ${ }^{1}$ S 37-44) wünschte man sich kürzer und prägnanter. Solons Wirken unter allen nur denkbaren Aspekten gelten die meisten Abschnitte dieses Kapitels. „2. Die Polis - Hüterin des sozialen Ausgleichs“ (BII ${ }^{1}$ S 59-76) bezieht sich ebenfalls auf Solon: Barta sieht in Solons Wirken „das Bemühen, einen Ausgleich zwischen den Polisbürgern herbeizuführen, ob reich oder arm, hochgestellt oder nicht“ (S 59). Dieser Feststellung kann man schwerlich widersprechen. Hinzu treten - nach Barta - weitere Momente, welche die klassische Polis ergäben. „Vorbilder für das von den Griechen früh und häufig eingesetzte Instrument 'Gesetz' und seine gesellschaftliche Funktion lieferten den Griechen wahrscheinlich Ägypten und Mesopotamien, vermittelt meist über den Vorderen Orient“" (S 61). Naturgemäß hat es allerorten an Rechtssetzung in Form königlicher Dekrete nicht gemangelt, denn Vielerlei bedurfte der Regelung. Hieraus auf allgemeinverbindliche, also gesetzliche Regelungen im heutigen Sinne zu schließen, wäre zweifellos verfehlt. Die Frage, inwieweit es in den Keilschriftrechten oder im pharaonischen Ägypten Gesetze gegeben hat, welche der griechischen Welt als Vorbild hätten dienen können, wird von Barta nicht eigens thematisiert. Eine andere, soweit ersichtlich anderwärts von Niemandem behandelte Frage hätte Barta bei seinem Vergleich vielleicht stellen können: Trat die inschriftliche Publikationsform der griechischen Gesetze als Autorisation an die Stelle der göttlichen Berufung der außergriechischen Herrscher?

Abschnitt 3 ist Drakon gewidmet (BII ${ }^{1} \mathrm{~S}$ 77-129) mit Unterabschnitten zur Person, zur Frage nach möglichen Vorbildern Drakons, dem möglichen Anlaß zu Drakons Tätigwerden, zu frühem Rechtsgang und Selbsthilfe, zur Entstehung des Schuldbegriffs und Einiges mehr. Die folgenden Abschnitte knüpfen daran an. B. umreißt die Entwicklung des Verschuldensprinzips und behandelt dazu Antiphon, Aristoteles und Anaximenes von Lampsakos sowie wiederum Drakon (,4. Das Entstehen der Rechtskategorie 'Zufall', S 130-212; ,5. Vom sakralen Sühnerecht zur säkularen Schuldlehre“, S 213-264; „6. Drakons Gesetz über die Blutrache“, S 265 292). „7. Wegweiser zur 'Eunomia'“ wendet sich wieder Solon zu und damit einem Thema, auf das B. dann in Abschnitt 17 (BII ${ }^{2}$ S 217-277) weiter eingeht. Solons Rechts- und Gerechtigkeitsvorstellungen sind hier ein vorrangiger Gegenstand der Erörterung. Unter „8. Menschliche Gerechtigkeit und göttliches Gesetz“ (BII ${ }^{1} \mathrm{~S}$ 311-317) verbindet Solons Wirken mit Gedanken von Hesiod. „9. Rechtssubjekt und Demokratie“ (BII ${ }^{1}$ S 318-441) weist die Entstehung des „Rechtssubjekts“ den

wirtschaftlichen und sozialen Gegebenheiten jener Epoche. Der zweite Hauptteil (S 213-464) ist Solons Leben und Wirken gewidmet. Siehe ferner JA Almeida Justice as an Aspect of the Polis Idea in Solon's Political Poems. A Reading of the Fragments in Light of the Reasearches of New Classical Archaeology (Leiden / Boston, 2003) [nv]; sowie neuerdings E Ruschenbusch Solon: Das Gesetzeswerk - Fragmente. Übersetzung und Kommentar (hrsgg von K Bringmann) (Stuttgart, $\left.{ }^{2} 2014\right)$. 
Griechen (mit bleibendem Nachwirken) zu. Hieran knüpft Barta seine eingehende Darstellung der damit veranlassten (oder diesbezüglich von Barta erörterten) gesellschaftlichen, wirtschaftlichen und demokratischen Verhältnisse. Hierzu gehört sE auch der Vertrag, und umfangreiche Erörterungen betreffen unter anderem das Synallagma, Schuld und Haftung, den griechischen Vertrag (S 374-438; su). Die meisten der folgenden Abschnitte dieses Kapitels gelten Solons Wirken unter weiteren denkbaren Aspekten. Teilweise drücken das bereits die Abschnittstitel aus: „10. Solons Gesetzgebung“ (BII ${ }^{1}$ S 442-606; mit Ausführungen zu frühen Gesetzgebern); „15. Solons Bild in der Geschichte“ (BII ${ }^{2}$ S 182-189) - „16. Solons Reformdenken“ (BII ${ }^{2}$ S 190-216; enthält viele durch Solon veranlaßte rechtspolitische Gedanken.) und weitere knüpfen an Solon an. Dies gilt sowohl für Bartas bereits erwähnte Ausführungen zu „13. Epieikeia“ (BII ${ }^{2}$ S 70-145) wie auch für „14. Hybrisklage und Persönlichkeitsschutz“ (BII ${ }^{2}$ S 146-189). Wiederum bietet Barta eine Fülle

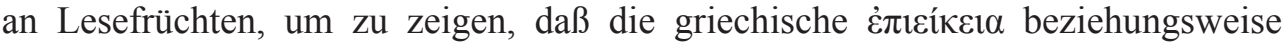
die Klage wegen Hybris Ansatzpunkte für bis heute wirksame Entwicklungen in Sachen „Billigkeit“ beziehungsweise „Persönlichkeitsrecht“ gewesen seien. Und wiederum verbieten es der hier verfügbare Raum wie Bartas detaillierter Inhalt, auf Bartas Darlegungen an dieser Stelle im Einzelnen einzugehen. In „17, 'Eunomia' und ägyptische 'Ma'at'، (BII' S 217-277) trägt B. seine Gedanken zum Thema vor, ob Solons Konzept der „Eunomia“ (Ev̉vo $\mu_{i ́ \alpha}$,gesetzlich geregelte Ordnung“ oä) ägyptisch beeinflußt sei. Nun könnte man freilich einwenden, daß die Belege zum ägyptischen Rechtsalltag der Pharaonenzeit von einer gelebten „Ma’at“ nichts erkennen lassen, aber das ist für philophische Gedanken der Normalfall, und falls für Solons Denken altägyptische Vorbilder in Betracht kämen, so entstammten diese keinesfalls dem Alltag. Über ein (wohlwollendes) non liquet kann man ungeachtet von Bartas Bemühen nicht hinauskommen. ${ }^{58}$ Auch in diesem Abschnitt exzerpiert Barta in hohem Maße Literaturmeinungen. Das gilt ebenso für die folgenden Abschnitte. „18. Das Stadtrecht von Gortyn“ (BII ${ }^{2}$ S 278-295) gibt einen unselbständigen Überblick über diese dem 5. Jahrh v Chr entstammende, höchst umfangreiche Inschrift mit rechtlichen Regelungen (um den Begriff „Gesetz“ zu vermeiden). Wie Barta Dokumentieren versteht, zeigt seine Erläuterung zur Auswahl der von ihm angegebenen Monographien und Darstellungen ,die wenigstens Ausschnitte des Stadtrechts bringen“ (S 279). Damit bleibt Vielerlei unerwähnt, wie Ausgaben des „Codex Gortyn“ zeigen, ebenso dessen Abdruck in einer anderweitigen neueren Inschriftensammlung und selbst Monographien. ${ }^{59}$ Zwischenzeitliche Inschriftenfunde bleiben gleichfalls unberücksichtigt.

58 Vgl dazu allerdings die BII² S 1420 Anm 1419/20 mit zugehörigem Text wiedergegebene Meinung von S Lippert aaO (oben Anm 6) S 2/3. Darauf kann hier nicht eingegangen werden.

59 SM Guarducci Inscriptiones Creticae, 1935-1950, Vol 4 (Rome, 1950); RF Willets The Law Code of Gortyn (Berlin, 1967); Nomima. Recueil d'inscriptions politiques et juridiques de l'archaïsme grec I; II (hrsgg von H van Effenterre \& F Ruzé (Rom, 1994 bzw 1995)); CE Gorlin The Gortyn 
In „12. Entstehung des Rechtssystems“ (S 46-69) skizziert Barta seine Sicht der Entwicklung der griechischen Rechtsordnung - detailreich und bei aller Kürze umfassend sowie losgelöst vom solonischen Wirken, allerdings mit vielen Verweisungen auf seine eigenen anderweitigen Ausführungen. ${ }^{60}$ In den Abschnitten „19. Vom Totenteil zum Individualeigentum“ (BII ${ }^{2}$ S 298-316), „20. Die Seelgerätestiftung“ (BII ${ }^{2}$ S 317-334) und „21. Hellenistische Totenkultstiftung - Römische Stiftungen - Germanisch-christliches 'Seelgerät'" (BII ${ }^{2}$ S. 335-343) sucht Barta anhand diverser Thesen des Rechtshistorikers EF Bruck die Entstehung des Privateigentums zu entwickeln. Seine Ausführungen enden folgerichtig in „22. Erwerb und Schutz von Individualeigentum“ (BII ${ }^{2}$ S 344-374), ein Abschnitt, der vor allem auf dem einschlägigen Werk von A Kränzlein beruht. ${ }^{61}$ Brucks Bedeutung für die Rechtsgeschichte aus Bartas Sicht machen eine Abbildung (341) und ein Unterabschnittstitel „Bruck - Mentor moderner Rechtsgeschichte“ (S 342) deutlich.

7 Einige weitere Beobachtungen sind anzuschließen. „Mit dem Entstehen des Rechtssubjekts ist ... die Fähigkeit verbunden, Träger eigener Rechte und Pflichten zu sein:Rechtsfähigkeit."Hierzu zählt Barta auch die Vertragsfreiheit (II $\left.{ }^{1}, \mathrm{~S} 360\right)$, und das rechtfertigt ihm, rechtsdogmatische Grundgedanken zum griechischen Vertragsrecht zu entwickeln (II ${ }^{1}$, S 360/374-440). Barta betont, ,unverzichtbare Grundlage des Vertragsschlusses" war auch in frühen Rechtsordnungen die Willenseinigung der Parteien ... (II $\left.{ }^{1}, \mathrm{~S} 376\right)$. Barta nimmt also an, daß ein Vertrag bereits mehr oder minder durch schlichten Konsens zustande gekommen ist. Selbst für das römische Recht trifft das so nicht zu, wie die Libralakte zeigen, und für andere Rechtsordnungen kann es nicht einfach vorausgesetzt werden. Natürlich bedarf es für den Abschluß eines Rechtsgeschäfts eines Grundkonsenses zwischen den Parteien, denn es kann zum Zustandekommen eines Vertrags nicht die eine Seite eine Eheschließung, die andere eine Pacht wollen. Dieser Grundkonsens besagt freilich nicht, daß ein Vertrag bei Übereinstimmung wirksam zustande kommt. Und hieraus ergibt sich die grundlegende Divergenz der Thesen Bartas beziehungsweise von HJ Wolff. Barta verficht den Vertragsschluß durch einen, ggf bezeugten Konsensualvertrag, der in durchaus modernem Sinn ein Leistungsversprechen bedeutet. Die von HJ Wolff entwickelte „Theorie der Zweckverfügung“ hingegen verneint ein solches

Law Code and Roman Crete (Diss PhD, Brown University, 1991); und Andere mehr.

60 Sie sind oft wenig hilfreich, vgl zB Anm BII 333 „Es ist bekannt, dass sich Rhetoren/Logographen früh spezialisiert haben; siehe bei Anm 417 mwH“ - Anm 417 „Rhetoren und Sophisten fördern neben erster Spezialisierung auch bereits Schulenbildung“ - Text dazu „Antiphon war ein Spezialist für Mord- und Totschlags-, Isaios für Erbrechtsfälle.“ - Das kann natürlich nur für die erhaltenen Reden gelten! - Gegen sich selbst spricht die Formulierung zu Verfügungen auf den Todesfall: „... als erster Schritt zu ihrer Entstehung dient die homerische donatio mortis causa iminente periculo ..." (BII $\left.{ }^{2} \mathrm{~S} 65\right)$.

61 A Kränzlein Eigentum und Besitz im griechischen Recht des fünften und vierten Jahrhunderts $v \operatorname{Chr}$ (Berlin, 1963). 
verbindliches Leistungsversprechen. ${ }^{62}$ Um an Wolffs Wortwahl anzuknüpfen, aber eine eigene Formulierung zu wählen: Eine - naturgemäß einverständliche - auf den Vertragszweck gerichtete (dingliche) Verfügung schafft eine Bindung zwischen den Parteien, kraft der der Verpflichtete haftet, sofern er das Zugesagte nicht leistet; seine Leistung ist also nicht die Erfüllung einer Schuld, sondern vermeidet die Realisierung der Haftung. Die Verfügung bringt die Verbindlichkeit zustande, sagt selbst aber über die Wirkung nichts aus; der Begriff, Verfügung“" spiegelt übrigens allerdings, soweit ich weiß, durchaus unbeabsichtigt - das von Wolff vorausgesetzte reale Element. ${ }^{63}$ Es überstiege den Rahmen jeder Besprechung, Bartas Einwände gegen Wolffs Vertragsthese hier widerzugeben und im Einzelnen zu würdigen oder auch nur gewisse Weiterentwicklungen der These zu schildern. Es ist ferner kein Argument, daß Wolffs Thesen von der heutigen juristischen Gräzistik allgemein akzeptiert sind - die Menge macht es nicht, aber auch nicht die Argumente früherer gräzistischer Rechtsliteratur. Wolffs „Theorie der Zweckverfügung“ entspricht offenbar dem Quellenbefund bestens. Bezüglich der Homologie (BII ${ }^{2}$ S 383-388)

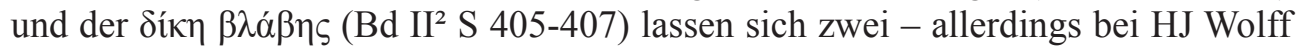
entstandene Untersuchungen - anführen. ${ }^{64}$ Wert legt Barta des Weiteren auf eine Dissonanz zwischen A Biscardi und HJ Wolff (BII² S 383-388). Eine gelegentliche Diskussion mit A Biscardi hat freilich seinerzeit ergeben, daß da offenbar ein sprachliches Mißverständnis vorgelegen hat. ${ }^{65}$

Barta erwähnt mehrfach das Verlöbnis. Als Zivilrechtler ist ihm bekannt, daß das Verlöbnis ein Vorvertrag ist, gerichtet auf das Eingehen des Hauptvertrags, der Eheschließung. Einen derartigen Vorvertrag hat es, soweit ersichtlich; weder in den Keilschriftrechten noch im ägyptischen, jüdischen oder griechischen Recht gegeben. Auch hier sei auf Nachweise verzichtet.

62 Grundlegend HJ Wolff „Die Grundlagen des griechischen Vertragsrechts“ in (1957) 74 Zeitschrift der Savigny Stiftung für Rechstgeschichte Rom Abt S 26-72 (abgedruckt in Zur griechischen Rechtsgeschichte (hrsg von E Berneker (Darmstadt, 1968) S 483-533).

63 Ein reales Element setzt auch E Seidl mit seinem „Prinzip der notwendigen Entgeltlichkeit“ voraus. Es hat zwar in der Tat wenig Befürworter gefunden. Das darf aber nicht der Anlaß sein, es mit diesem Hinweis abzutun und nicht einmal eine Fundstelle zu nennen; so aber BII ${ }^{2} \mathrm{~S} 381$ Anm 2294. Zu dem genannten Prinzip siehe beispielsweise E Seidl Aegyptische Rechtsgeschichte der Saiten- und Perserzeit (Glückstadt / Hamburg / New York, ${ }^{2} 1968$ ) S 45-50. Vgl dazu T Mrsich aaO (I, o Anm 6) S 513/4 mit Anm 14.

64 Zur Homologie (siehe BII ${ }^{2}$ S 376, Anm 2259) siehe H von Soden Untersuchungen zur Homologie in den griechischen Papyri Ägyptens bis Diokletian (Köln / Wien, 1973); vgl dazu (ua) die

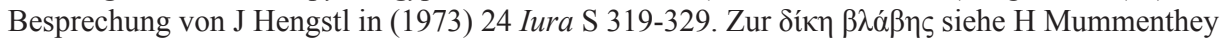
Zur Geschichte des Begriffs $\beta \lambda \alpha ́ \beta \eta$ im attischen Recht (Jur Diss, Freiburg, 1971).

65 Siehe J Hengstl, Besprechung von Symposion 1974, Vorträge zur griechischen und hellenistischen Rechtsgeschichte (Gargnano am Gardasee, 5.-8. Juni 1974) in Gemeinschaft mit HJ Wolff, J Modrzejewski \& P Dimakis herausgg von A Biscardi (Köln / Wien, 1979) in (1980) 97 Zeitschrift der Savigny Stiftung für Rechstgeschichte Rom Abt S 348-355 (349-350). 
Methodisch nicht $\mathrm{zu}$ befriedigen vermag die Verwendung der attischen Gerichtsreden. Barta behandelt sie gewöhnlich als statische Belege des griechischen Rechtsdenkens. Tatsächlich aber sind die erhaltenen Gerichtsreden weniger Zeugnisse der Rechtsordnung als der rhetorischen Kunst. Die überlieferten Reden entsprechen einem Kanon, der vor der Mitte des 1. Jahrh v Chr erstellt worden ist und die als die zehn Besten ihrer Zeit erachteten Redner auflistet. Keineswegs alle stammen von den Redenschreibern, denen sie zugeschrieben sind. Doch hierauf kommt es für die Rolle der Reden als Rechtszeugnisse nicht an. Die Gerichtsreden waren zum Vortrag vor dem dikasterion bestimmt und entsprachen dessen Verfahren. Die Richterbänke waren allein mit Laienrichtern besetzt, welche ohne Abstimmung ihr Urteil abgaben. Es galt, diese Richter durch den Vortrag zu überzeugen. Diesen prägten daher vor allem rhetorische Mittel. Unter diesem Gesichtspunkt ist auch die Interpretation von Gesetzen im attischen Prozeß zu sehen, und das ist zu berücksichtigen, wenn man attische Gerichtsreden als Rechtsquellen heranzieht. ${ }^{66}$

8 Bartas monumentales Werk ist entgegen allem Anschein weder eine Geschichte des griechischen Rechts noch eine Geschichte der griechischen Rechtsgeschichte als Wissenschaftsdisziplin. Sein wesentliches Anliegen, den Fluß der Rechtsentwicklung von der Antike bis nachgerade heute zu schildern, entspricht keinem von Beidem. Seine Darstellungsweise verdeckt eine Fülle an bemerkenswerten Gedanken und Anregungen. Nur: Wie soll man diese unter der Unzahl an übernommenen Texten finden? Sie verdecken zudem Bartas eigene Gedankengänge. Diese sind nämlich oftmals kaum von den die Textübernahmen verbindenden Worten zu unterscheiden oder diesen zu entnehmen. Immer wieder gibt es eine Vielzahl an Ausführungen, Textübernahmen und EntwicklungsSchilderungen, die einer eingehenderen Diskussion und der Darstellung von Gegenmeinungen bedürften - zu viele als daß sie hier auch nur aufgezählt werden könnten. Die graphische Gestaltung (Wechsel von Normal zu Petit) trägt dazu keinesfalls bei. Es liegt schließlich ferner auf der Hand, daß die Fülle der wörtlichen Übernahmen die eigene Analyse und Exegese nicht zu ersetzen vermag. Reduziert auf das dem Thema Wesentliche, bar aller fremder Texte, die eigenen Gedanken anhand der exegierten und analysierten Primär- und Sekundärquellen vortragend,

66 Siehe dazu M Hillgruber Die zehnte Rede des Lysias: Einleitung, Text und Kommentar mit einem Anhang über die Gesetzesinterpretationen bei den attischen Rednern (Berlin / New York, 1988) S 105-120; ferner HJ Wolff „Methodische Grundfragen der rechtsgeschichtlichen Verwendung attischer Gerichtsreden" in La critica del testo. Atti del II Congresso Internazionale della Società Italiana di Storia del Diritto, Venezia, 18 - 22 Settembre 1967 (Florenz, 1971) Vol 2 S 1123-1135 (abgedruckt in HJ Wolff Opuscula dispersa (Amsterdam, 1974) S 27-39); Dens Demosthenes als Advokat. Funktionen und Methoden des Prozesspraktikers im klassischen Athen. Vortrag gehalten vor der Berliner Juristischen Gesellschaft am 30 Juni 1967 (Berlin, 1968) und abgedruckt in Demosthenes hrsgg von U Schindel (Darmstadt, 1977) S 376-402. 
hätte Barta ein für die antike Rechtsgeschichte außerordentlich wichtiges Werk geschaffen.

\section{Nachtrag}

$\mathrm{H}$ Barta hat mit seinem monumentalen, auf vier Bände angelegten Werk „Graeca non leguntur" wichtige, aber oft anfechtbare Denkanstöße zum griechischhellenistischen Recht und zu dessen Auswirkungen gegeben. Nunmehr legt er das erste Buch des wiederum zweifach unterteilten Bandes III (BIII ${ }^{1}$ ) vor. ${ }^{67}$ Der Band irritiert auf den ersten Blick. Seine Gliederung ist mit „Zweiter Teil: Dichtung, Geschichte, Philosophie und Recht“ überschrieben. Ein „Erster Teil“ ist nirgendwo ausgewiesen und kann wohl nur den Inhalt von Band 1 und/oder Band 2 meinen. Darüber hinaus vermißt man den laut $\mathrm{BII}^{2}$, S 375 aus zeitlichen Gründen nach Band 3 verwiesenen letzten Abschnitt von Kapitel 2, Abschnitt 23 „Rezeption und Kulturtransfer aus dem Alten Orient". Aufbau und Vorgehensweise von Band $3^{1}$ entsprechen den vorausgegangenen Bänden. Den üblichen Präliminarien (S I-XXI) folgen die Einleitung (S 1-25) und die Kapitel 3 („Die ‘Eumeniden’ des Aischylos“; S 34-164), 4 („Der 'Melierdialog' des Thukydides“; S165-219) und 5 („Euripides und das Naturrecht"; S 220-326). Glossar (S 327-393); Literaturverzeichnis (S 395-522), Stellenregister (S 523-529) und Stichwörter (S 530-547) beschließen den Band. Die Tatsache, daß ein mit jedem Band wachsender Anteil dieser Anhänge nicht nur Platz, sondern auch Aufmerksamkeit des Benutzers beansprucht, läßt Barta’s bandweisen Aufbau der Nachweise verwerfen.

Der Inhalt von Band 3 ist mit dem Untertitel des Bandes bestens umrissen: Es geht um „Das griechische Recht in seinem kulturhistorischen Umfeld - Beispiele aus Dichtung, Geschichtsschreibung, Philosophie und (Kautelar) Jurisprudenz". Zur Kautelarjurisprudenz, also zu Alltagsurkunden, findet sich, wie auch das Stellenregister ausweist, nichts. Die in den Kapitelüberschriften Genannten Aischylos, Thukydides und Euripides - sind auch von E Wolf in seiner Darstellung des griechischen Rechtsdenkens berücksichtigt worden. ${ }^{68}$ Es ist bezeichend für die unterschiedlich Betrachtungsweise von Barta und Wolf, daß Barta auf Wolf (den Anmerkungen nach zu schließen) überhaupt nicht eingeht. Bartas Vorgehensweise entspricht der in den vorausgehenden Bänden: Barta referiert allzu viel Lesefrüchte, statt in eigenen Ausführungen auf andere Meinungen als Belege zu verweisen; seine Fußnoten sind voll von Bemerkungen und Anspielungen, die zum Thema mal mehr,

67 H Barta 'Graeca non leguntur'? - Zu den Ursprüngen des europäischen Rechts im antiken Griechenland. Band III/1: Das griechische Recht in seinem kulturhistorischen Umfeld-Beispiele aus Dichtung, Geschichtsschreibung, Philosophie und (Kautelar) Jurisprudenz (Wiesbaden, 2014) ISBN 978-3-447-10036-6 Gr 8; XXI 547 S.

68 E Wolf Griechisches Rechtsdenken 4 in 6 Banden (Frankfurt/M, 1950-1970). Zu Aischylos siehe besonders Bd 1 S 340-424; zu Euripides siehe besonders Bd 2 S 373-481. Als Geschichtsschreiber findet Thukydides bei Wolf keine besondere Berücksichtigung. 
mal gar nichts beitragen. Allerdings ist zu vermerken, daß Barta stärker als in den vorhergehenden Bänden seine eigene Meinung zu Wort kommen läßt. Es scheint, als habe Barta hier eine ihm besonders wesensnahe Thematik behandelt. Das umfaßt eine Fülle an eher rechtsphilosophisch einzustufenden Ausführungen. Sie sind ebenso interessant wie diskussionswürdig und enthalten immer wieder rechtshistorisch relevante Topoi. Allerdings macht Barta es dem Leser selbst in diesem Band schwer, das rechtshistorisch Wesentliche nachzuvollziehen. $\mathrm{Zu}$ vermischt sind seine diversen Gesichtspunkte und Argumentationen. Man muß sie lesen, und das gilt auch für die zahllosen Anmerkungen. Aber die Lektüre ist zweifellos ein Gewinn, wenn auch weniger für die rechtsgeschichtliche Darstellung und Diskussion.

\section{Abstract}

The ancient world saw the rise and fall of many cultures, with accompanying cultural exchanges and reciprocal influences. It seems that such reciprocal influences and exchanges extended to the law as well, and affected legal evolution. Old legal institutions were preserved for future generations but were influenced by newer cultures. This concept is not new, but Barta has revived it. He has embarked on a publication comprising four volumes, of which volumes $1,2(\mathrm{pt} 1 / 2)$ and 3 (pt 1) have appeared. The author has set out to prove that the influence of Greek law on Roman law was greater than has been acknowledged up until now, that Greek law was likewise influenced by the Old Orient, and that Roman law also influenced later legal systems. The one concern of this article is to show that Barta's theory is invalid. The other concern is to criticise Barta's approach. He does not cite the extensive literature on which he relies, but quotes it. He fills page after page with texts by other authors but does not discuss nor analyse their content. Thus the reader is provided with a wide range of legal literature of the past, but not with any critical analysis of it. Newer writing is often neglected. Barta himself has interesting ideas, but it is difficult to detect them, hidden as they are amongst the cited literature. This approach is followed in all volumes but is scientifically unacceptable and merits the strongest criticism. 